KIAS-P10034

\title{
Interaction between M2-branes and Bulk Form Fields
}

\author{
Yoonbai Kim ${ }^{1}$, O-Kab Kwon ${ }^{1}$, Hiroaki Nakajima ${ }^{3,4}$, D. D. Tolla ${ }^{1,2}$ \\ ${ }^{1}$ Department of Physics, BK21 Physics Research Division, Institute of Basic Science, \\ ${ }^{2}$ University College, \\ Sungkyunkwan University, Suwon 440-746, Korea, \\ ${ }^{3}$ School of Physics, Korea Institute for Advanced Study, Seoul 130-722, Korea, \\ ${ }^{4}$ Department of Physics, Kyungpook National University \\ Taegu, 702-701, Korea \\ yoonbai@skku.edu, okab@skku.edu, nakajima@kias.re.kr, ddtolla@skku.edu
}

\begin{abstract}
We construct the interaction terms between the world-volume fields of multiple M2branes and the 3- and 6-form fields in the context of ABJM theory with $\mathrm{U}(N) \times \mathrm{U}(N)$ gauge symmetry. A consistency check is made in the simplest case of a single M2-brane i.e., our construction matches the known effective action of M2-brane coupled to antisymmetric 3 -form field. We show that when dimensionally reduced, our couplings coincide with the effective action of D2-branes coupled to R-R 3- and 5-form fields in type IIA string theory. We also comment on the relation between a coupling with a specific 6-form field configuration and the supersymmetry preserving mass deformation in ABJM theory.
\end{abstract}




\section{Contents}

1 Introduction $\quad 2$

2 Couplings between M2-branes and Form Fields 4

3 Consistency Check for Single M2-brane

4 Reduction from M-theory to IIA String Theory 9

$4.1 \mathrm{U}(1) \times \mathrm{U}(1)$ gauge symmetry . . . . . . . . . . . . . . . . . . . . . . . 9

$4.2 \mathrm{U}(N) \times \mathrm{U}(N)$ gauge symmetry . . . . . . . . . . . . . . . . . . . . . . . . 13

$4.2 .1 \quad 3$-form fields . . . . . . . . . . . . . . . . . . . . 14

$4.2 .2 \quad 6$-form fields . . . . . . . . . . . . . . . . . . 19

5 Comments on Mass Deformations $\quad 24$

6 Conclusion and Discussion $\quad 25$

\begin{tabular}{|l|l|}
\hline A Comments on Single Trace Terms & 27 \\
\hline
\end{tabular}

\section{Introduction}

Recently, the Lagrangian descriptions of multiple M2-branes were found in the low energy limit, which are the Bagger-Lambert-Gustavsson(BLG) theory [1, 2] and the Aharony-Bergman-JafferisMaldacena(ABJM) theory [3]. The BLG theory, which is equivalent to the ABJM theory with $\mathrm{SU}(2) \times \mathrm{SU}(2)$ gauge group [4], has $\mathcal{N}=8$ supersymmetry and it is an effective theory of two M2-branes. The ABJM theory with $\mathrm{U}(N) \times \mathrm{U}(N)$ gauge group has $\mathcal{N}=6$ supersymmetry and it describes the dynamics of $N$ parallel M2-branes sitting at the singularity of a space with $\mathbb{Z}_{k}$ orbifold, where $k$ appears as the Chern-Simons level in the theory.

Low energy dynamics of D-branes is also depicted by supersymmetric gauge theories, i.e., it is the Dirac-Born-Infeld(DBI) action or the super Yang-Mills theory to the leading order in $\alpha^{\prime}$ expansion. In addition, D-branes can couple to the bulk supergravity fields. The bosonic bulk fields include R-R form fields, which couple to the D-branes through Wess-Zumino(WZ)-type action [5, 6, 7]. In the case of single $\mathrm{D} p$-brane, the WZ-type coupling is only to the R-R form fields of rank $p+1$ or less. For multiple $\mathrm{D} p$-branes the action can include the couplings to all kinds of R-R form fields [7]. 
Analogous to the WZ-type coupling of D-branes in string theory, in M-theory, the M2- and M5-branes couple to the 3-form and dual 6-form fields in 11-dimensional supergravity. According to the recent development of the world-volume theories for multiple M2-branes, the coupling of M2-branes to the bulk form fields were discussed in the context of both the BLG theory [8, 9, 10] and the ABJM theory [11, 12. For arbitrary $N$ stacked M2-branes the analyses have been made in the restricted context: Ref. [11] dealt with the coupling with constant form fields which survive in the infinite tension limit of M2-branes and Ref. [12] considered only the case of single M2-brane. Along this line it is timely to study the coupling of $N$ M2-branes to the bulk form fields which have arbitrary dependence on the transverse scalar fields.

In our previous paper [10], we discussed the coupling between M2-branes and the bulk form fields in BLG theory with the aforementioned general setting. After the compactification procedure of Mukhi-Papageorgakis (MP Higgsing procedure) [13, 14], we verified that our proposal reproduces the $\mathrm{R}$ - $\mathrm{R}$ form field couplings to D2-branes as well as the linearized DBI action in type IIA string theory. In this paper we naturally generalize our previous construction to multiple M2-branes of arbitrary $N$, described by the ABJM theory with $\mathrm{U}(N) \times \mathrm{U}(N)$ gauge symmetry. Guided by gauge invariance, we propose a general form of WZ-type coupling. In our proposal the form fields can carry gauge indices since we assume that the form fields depend on the transverse scalar fields in (anti)bifundamental representations of the gauge group. Since M-theory provides no direct guiding principle for the WZ-type coupling, there seems no compelling reason to restrict the form fields to be antisymmetric in the global indices. Reflecting consistency with the known WZ-type couplings of type IIA string theory after the MP Higgsing, one can constrain the Mtheory form fields to appropriate forms. To be specific, we impose some appropriate constraints to the 3- and 6-form fields in M-theory and obtain the relations among these form fields and the R-R and NS-NS form fields in type IIA string theory. These constraints lead to the antisymmetric property of the form fields and the symmetrized matrix products in the resulting WZ-type couplings [7] of type IIA string theory. A byproduct is the generation of the linearized DBI action of multiple D2-branes, where the gauge field strength $\tilde{F}_{\mu \nu}$ and the NS-NS 2-form field $\tilde{B}_{\mu \nu}$ appear in the gauge-covariant combination, $\tilde{F}_{\mu \nu}+\frac{1}{2 \pi \alpha^{\prime}} \tilde{B}_{\mu \nu}$.

The MP Higgsing for the WZ-type coupling of the 3-form fields is completely carried out, however the corresponding procedure for the case of the 6-form fields is performed only up to the quadratic order terms in Planck length. For the cubic order, there are too many possible terms which appear in the MP Higgsing procedure and this makes the derivation of explicit relations between the R-R 5-form fields and the 6-form fields complicated. We do not carry out the reduction procedure for this order terms, rather we guess the expected couplings from the results of the lower order terms in Planck length. 
This paper is organized as follows. In section 2, we propose the general structure of the coupling between multiple M2-branes and the bulk form fields. In section 3, we compare the proposal with the well known coupling for the case of a single M2-brane. In section 4, we check our proposal by taking the reduction to type IIA string theory, via the MP Higgs mechanism. In section 5, we establish the relation between our WZ-type coupling and the supersymmetry preserving mass deformation in ABJM theory. Section 6 is devoted to conclusion and discussion.

\section{Couplings between M2-branes and Form Fields}

Low energy effective world-volume theory of the stacked $N$ parallel flat M2-branes is described by the ABJM action. Its bosonic sector contains two gauge fields, $A_{\mu}$ and $\hat{A}_{\mu}$, of $\mathrm{U}(N) \times \mathrm{U}(N)$ gauge symmetry and four complex scalar fields $Y^{A},(A=1,2,3,4)$. The real and imaginary components of $Y^{A}$ represent the eight transverse directions $X^{I},(I=1,2, \ldots, 8)$,

$$
Y^{A}=X^{A}+i X^{A+4} .
$$

The scalar fields are in the bifundamental representation of the $\mathrm{U}(N) \times \mathrm{U}(N)$ gauge symmetry while the gauge fields $A_{\mu}$ and $\hat{A}_{\mu}$ are in the adjoint representations of the left $\mathrm{U}(N)$ and the right $\mathrm{U}(N)$ gauge symmetries, respectively. Including the gauge indices we can write the bosonic fields as

$$
\left(A_{\mu}\right)_{b}^{a}, \quad\left(\hat{A}_{\mu}\right)_{\hat{b}}^{\hat{a}}, \quad\left(Y^{A}\right)_{\hat{a}}^{a} \quad\left(Y_{A}^{\dagger}\right)_{a}^{\hat{a}} .
$$

Low energy effective description of M-theory is known to be the 11-dimensional supergravity for which the bosonic fields are graviton $g_{M N}, 3$-form $C_{(3)}$, and 6-form $C_{(6)}$ fields. Those form fields can interact with the world-volume fields through pull-back. The corresponding interactions in 10-dimensional type II string theories are described by WZ-type couplings [5, 6, 7]. However, in the scheme of M-theory, the form of such interaction is not yet known except in BLG theory with $\mathrm{SU}(2) \times \mathrm{SU}(2)$ gauge symmetry [8, 10] and ABJM theory with constant form fields [11]. In this section we naturally extend the results in [10] to the case of ABJM theory with $\mathrm{U}(N) \times \mathrm{U}(N)$ gauge symmetry.

We start with a general gauge-invariant couplings of 3-form fields to multiple M2-branes. In analogy with the WZ-type couplings in type II string theories we restrict the couplings to terms which are linear in the form fields. Our proposal for the 3-form fields action is

$$
\begin{aligned}
S_{C}^{(3)}=\mu_{2} \int d^{3} x & \frac{1}{3 !} \epsilon^{\mu \nu \rho}\{\operatorname{Tr}\}\left[C_{\mu \nu \rho}+3 \lambda C_{\mu \nu A} D_{\rho} Y^{A}+3 \lambda^{2}\left(C_{\mu A B} D_{\nu} Y^{A} D_{\rho} Y^{B}+C_{\mu A \bar{B}} D_{\nu} Y^{A} D_{\rho} Y_{B}^{\dagger}\right)\right. \\
& \left.\left.+\lambda^{3}\left(C_{A B C} D_{\mu} Y^{A} D_{\nu} Y^{B} D_{\rho} Y^{C}+C_{A B \bar{C}} D_{\mu} Y^{A} D_{\nu} Y^{B} D_{\rho} Y_{C}^{\dagger}\right)+\text { (c.c. }\right)\right]
\end{aligned}
$$


where $\mu_{2}$ is M2-brane tension, $\lambda=2 \pi l_{\mathrm{P}}^{3 / 2}$ with Planck length $l_{\mathrm{P}}$, and $\{\mathrm{Tr}\}$ represents all possible contractions of gauge indices among the 3 -form fields and transverse scalars, which give single traces 1 The covariant derivative is

$$
D_{\mu} Y^{A}=\partial_{\mu} Y^{A}+i A_{\mu} Y^{A}-i Y^{A} \hat{A}_{\mu}
$$

In each term the dependence of the pull-back of the 3-form field on $Y^{A}$ and $Y_{A}^{\dagger}$ should be chosen in such a way that the term contains equal number of bifundamental and antibifundamental fields so that the action keeps manifest gauge invariance. The gauge invariance is achieved if we assume that the pull-back of the form fields can have multiple non-Abelian gauge indices which are allowed only through their functional dependence on the transverse scalars. This assumption is motivated by the analogy with $\mathrm{R}$ - $\mathrm{R}$ form field couplings to multiple $\mathrm{D} p$-branes in string theory [7], where the pull-back of the R-R fields are defined by just replacing the transverse coordinates with the transverse scalar fields. In the latter case, the dependence of the transverse scalars in the the pull-back of the R-R fields was obtained by performing the non-Abelian Taylor expansion [7]. For instance for a rank $n$ R-R form field it can be written as

$$
P\left(\tilde{C}_{(n)}\right)(\tilde{X})=\tilde{C}_{(n)}(0)+\tilde{X}^{i} \partial_{i} \tilde{C}_{(n)}(0)+\frac{1}{2 !} \tilde{X}^{i} \tilde{X}^{j} \partial_{i} \partial_{j} \tilde{C}_{(n)}(0)+\cdots
$$

where the product among $\tilde{X}^{i}$ 's is a matrix product. The expansion contains uncontracted gauge indices and, in the coupling between the $\mathrm{D} p$-branes and the $\mathrm{R}$-R fields, the gauge trace is taken including the pull-backed R-R form fields. This procedure was confirmed to leading order by examining string scattering amplitudes [15].

In order to clarify the meaning of $\{\mathrm{Tr}\}$ we present some terms in the action (2.3) including the gauge indices explicitly,

$$
\begin{aligned}
\{\operatorname{Tr}\}\left(C_{\mu \nu A} D_{\rho} Y^{A}\right) & =\left(C_{\mu \nu A}\right)_{a}^{\hat{a}}\left(D_{\rho} Y^{A}\right)_{\hat{a}}^{a}, \\
\{\operatorname{Tr}\}\left(C_{\mu A B} D_{\nu} Y^{A} D_{\rho} Y^{B}\right) & =\left(C_{\mu A B}\right)_{a b}^{\hat{a} \hat{b}}\left(D_{\nu} Y^{A}\right)_{\hat{a}}^{a}\left(D_{\rho} Y^{B}\right)_{\hat{b}}^{b}, \\
\{\operatorname{Tr}\}\left(C_{\mu A \bar{B}} D_{\nu} Y^{A} D_{\rho} Y_{B}^{\dagger}\right) & =\left(C_{\mu A \bar{B}}\right)_{a \hat{b}}^{\hat{a} b}\left(D_{\nu} Y^{A}\right)_{\hat{a}}^{a}\left(D_{\rho} Y_{B}^{\dagger}\right)^{\hat{b}}{ }_{b}, \\
\{\operatorname{Tr}\}\left(C_{A B \bar{C}} D_{\mu} Y^{A} D_{\nu} Y^{B} D_{\rho} Y_{C}^{\dagger}\right) & =\left(C_{A B \bar{C}}\right)_{a b \hat{c}}^{\hat{a} \hat{c} c}\left(D_{\mu} Y^{A}\right)_{\hat{a}}^{a}\left(D_{\nu} Y^{B}\right)_{\hat{b}}^{b}\left(D_{\rho} Y_{C}^{\dagger}\right)^{\hat{c}}, \\
\{\operatorname{Tr}\}\left(C_{A B C} D_{\mu} Y^{A} D_{\nu} Y^{B} D_{\rho} Y^{C}\right) & =\left(C_{A B C}\right)_{a b c}^{\hat{a} \hat{b} \hat{c}}\left(D_{\mu} Y^{A}\right)_{\hat{a}}^{a}\left(D_{\nu} Y^{B}\right)_{\hat{b}}^{b}\left(D_{\rho} Y^{C}\right)^{c} .
\end{aligned}
$$

In order to understand this gauge index structure let us take into account the Taylor expansion for the pull-backed form fields in the context of M-theory. Due to the gauge invariance and the

\footnotetext{
${ }^{1}$ In our convention the unbarred global indices $A, B, C, \cdots$ of the form fields are contracted with those of bifundamental scalar fields while the barred global indices $\bar{A}, \bar{B}, \bar{C}, \cdots$ are contracted with those of antibifundamental scalar fields. The unbarred indices are always located to the left of the barred ones.
} 
single traceness of the corresponding coupling, possible terms in the Taylor expansion for the form fields are restricted. As an example, we expand $C_{\mu A \bar{B}}$ in terms of the transverse scalar fields,

$$
\left(C_{\mu A \bar{B}}\right)_{a \hat{b}}^{\hat{a} b}=C_{\mu A \bar{B}}(0) \delta_{\hat{b}}^{\hat{a}} \delta_{a}^{b}+\partial_{\bar{C}} \partial_{D} C_{\mu A \bar{B}}(0)\left(Y_{C}^{\dagger} Y^{D}\right)_{\hat{b}}^{\hat{a}} \delta_{a}^{b}+\partial_{C} \partial_{\bar{D}} \hat{C}_{\mu A \bar{B}}(0) \delta_{\hat{b}}^{\hat{a}}\left(Y^{C} Y_{D}^{\dagger}\right)_{a}^{b}+\cdots
$$

where the form fields and their derivatives are functions of the worldvolume coordinates but do not depend on the transverse scalar fields. After being pulled-backed to the worldvolume, uncontracted gauge indices appear in the right-hand side of (2.7) due to their dependence on the transverse scalar fields. This clearly verifies that Taylor expansion of the form fields generates non-Abelian structure even though the bulk form fields themselves do not carry non-Abelian gauge indices. The same procedure can be applied for the other pull-backed 3- and 6-form fields in order to understand the non-Abelian gauge index structure.

From now on let us turn to the WZ-type coupling between M2-branes and 6-form fields. Along the same line with the 3 -form coupling, imposing the gauge invariance and allowing only single trace couplings, the most general 6-form field coupling which is linear in the 6-form fields is given by

$$
\begin{aligned}
& S_{C}^{(6)}=\mu_{2}^{\prime} \int d^{3} x \frac{1}{3 !} \epsilon^{\mu \nu \rho}\{\operatorname{Tr}\}\left(C_{\mu \nu \rho A B \bar{C}} \beta_{C}^{A B}+3 \lambda\left(C_{\mu \nu A B C \bar{D}} D_{\rho} Y^{A} \beta_{D}^{B C}+C_{\mu \nu A B \bar{C} \bar{D}} D_{\rho} Y_{C}^{\dagger} \beta_{D}^{A B}\right)\right. \\
& +3 \lambda^{2}\left(C_{\mu A B C D \bar{E}} D_{\nu} Y^{A} D_{\rho} Y^{B} \beta_{E}^{C D}+C_{\mu A B C \bar{D} \bar{E}} D_{\nu} Y^{A} D_{\rho} Y_{D}^{\dagger} \beta_{E}^{B C}+C_{\mu A B \bar{C} \bar{D} \bar{E}} D_{\nu} Y_{C}^{\dagger} D_{\rho} Y_{D}^{\dagger} \beta_{E}^{A B}\right) \\
& +\lambda^{3}\left(C_{A B C D E \bar{F}} D_{\mu} Y^{A} D_{\nu} Y^{B} D_{\rho} Y^{C} \beta_{F}^{D E}+C_{A B C D \bar{E} \bar{F}} D_{\mu} Y^{A} D_{\nu} Y^{B} D_{\rho} Y_{E}^{\dagger} \beta_{F}^{C D}\right. \\
& \left.\left.+C_{A B C \bar{D} \bar{E} \bar{F}} D_{\mu} Y^{A} D_{\nu} Y_{D}^{\dagger} D_{\rho} Y_{E}^{\dagger} \beta_{F}^{B C}+C_{A B \bar{C} \bar{D} \bar{E} \bar{F}} D_{\mu} Y_{C}^{\dagger} D_{\nu} Y_{D}^{\dagger} D_{\rho} Y_{E}^{\dagger} \beta_{F}^{A B}\right)+(\text { c.c. })\right),
\end{aligned}
$$

where $\mu_{2}^{\prime}=\tau \lambda \mu_{2}, \tau$ is a dimensionless parameter which will be fixed after reduction to type IIA string theory, and $\beta_{C}^{A B} \equiv \frac{1}{2}\left(Y^{A} Y_{C}^{\dagger} Y^{B}-Y^{B} Y_{C}^{\dagger} Y^{A}\right)$. We assumed that apart from their appearance in the covariant derivatives, the transverse complex scalar fields explicitly enter the action only in terms of this specific cubic product $\beta_{C}^{A B}$ and its complex conjugate. This assumption is motivated by the fact that $\beta_{C}^{A B}$ and its complex conjugate appear in the sextic potential of ABJM theory. Actually $\beta_{C}^{A B}$ corresponds to three commutator in hermitian 3-algebra formulation of the ABJM theory [16].

For every term in the action (2.8) the contraction of gauge indices is performed as was done for the 3 -form field coupling in (2.6) . For instance,

$$
\begin{aligned}
& C_{A B \bar{C} \bar{D} \bar{E} \bar{F}} D_{\mu} Y_{C}^{\dagger} D_{\nu} Y_{D}^{\dagger} D_{\rho} Y_{E}^{\dagger} \beta_{F}^{A B} \\
& =\left(C_{A B \bar{C} \bar{D} \bar{E} \bar{F}}\right)_{\hat{a} \hat{b} \hat{c} d}^{a b \hat{d}}\left(D_{\mu} Y_{C}^{\dagger}\right)_{a}^{\hat{a}}\left(D_{\nu} Y_{D}^{\dagger}\right)_{b}^{\hat{b}}\left(D_{\rho} Y_{E}^{\dagger}\right)_{c}^{\hat{c}}\left(\beta_{F}^{A B}\right)_{\hat{d}}^{d} .
\end{aligned}
$$

Furthermore we should restrict the form field as in (2.7) in order to obtain single trace couplings. 
It is not easy to show that the actions in (2.3) and (2.8) are invariant under gauge transformation of the 3- and 6-form fields $C_{(n)} \rightarrow C_{(n)}+d \Lambda_{(n-1)}$ due to the non-Abelian structure of the coupling. As was proven in the case of R-R fields in string theory [17, 18], this issue of gauge invariance will be addressed through further study [19].

\section{Consistency Check for Single M2-brane}

In this section we will test our proposal in the previous section by comparing with the well known effective action of a single M2-brane in the presence of 3-form field $\hat{C}_{m n p}$. The effective action is given by [20]

$$
S_{11}=-\mu_{2} \int d^{3} \sigma \sqrt{-\operatorname{det}\left(\partial_{\mu} x^{m} \partial_{\nu} x^{n} g_{m n}\right)}+\frac{\mu_{2}}{3 !} \int d^{3} \sigma \epsilon^{\mu \nu \rho} \hat{C}_{m n p} \partial_{\mu} x^{m} \partial_{\nu} x^{n} \partial_{\rho} x^{p},
$$

where $x^{m}(m, n, p=0,1,2, \ldots, 10)$ are the 11 -dimensional spacetime coordinates, $\sigma^{\mu}$ are the 3 dimensional world-volume coordinates, and $g_{m n}$ is 11-dimensional metric. For a single static flat M2-brane in flat $(1+2)$-dimensional spacetime and in an $\mathbb{R}^{8} / \mathbb{Z}_{k}$ orbifold for the transverse space, we locally have $g_{m n}=\eta_{m n}$. Choosing a static gauge $\sigma^{\mu}=x^{\mu}$ and regarding the 8 transverse coordinates as the 8 transverse scalar fields $x^{I}=\lambda X^{I}$, the 3 -form field coupling in (3.1) can be rewritten as

$$
\frac{\mu_{2}}{3 !} \int d^{3} x \epsilon^{\mu \nu \rho}\left(\hat{C}_{\mu \nu \rho}+3 \lambda \hat{C}_{\mu \nu I} \partial_{\rho} X^{I}+3 \lambda^{2} \hat{C}_{\mu I J} \partial_{\nu} X^{I} \partial_{\rho} X^{J}+\lambda^{3} \hat{C}_{I J K} \partial_{\mu} X^{I} \partial_{\nu} X^{J} \partial_{\rho} X^{k}\right)
$$

If we compare the obtained action (3.2) with the action (2.3) and (2.8) for a single M2-brane, they look different at first glance. However, we will show that one exactly coincides with the other. We can also show that (3.2) is invariant under the Abelian gauge transformation of 3-form field as $C_{(3)} \rightarrow C_{(3)}+d \Lambda_{(2)}$.

First of all, if we restrict ourselves to the $\mathrm{U}(1) \times \mathrm{U}(1)$ case of the actions (2.3) and (2.8),$\{\operatorname{Tr}\}$ becomes trivial due to the Abelian nature. Noticing that the complex scalar fields commute with each other, all the terms involving the 6 -form field are vanishing. Therefore the total action with $\mathrm{U}(1) \times \mathrm{U}(1)$ symmetry is obtained only from the action (2.3). Secondly, we note that the action (2.3) involves $\mathrm{U}(1) \times \mathrm{U}(1)$ gauge fields, while the action (3.2) does not. Dynamics of the gauge fields is governed by the Chern-Simons action in the ABJM theory,

$$
S_{\mathrm{CS}}=\int d^{3} x \frac{k}{4 \pi} \epsilon^{\mu \nu \rho}\left(A_{\mu} \partial_{\nu} A_{\rho}-\hat{A}_{\mu} \partial_{\nu} \hat{A}_{\rho}\right),
$$

and the matter fields couple to the gauge fields only through the covariant derivative (2.4). If we introduce $A_{\mu}^{ \pm}$as

$$
A_{\mu}^{ \pm}=\frac{1}{2}\left(A_{\mu} \pm \hat{A}_{\mu}\right)
$$


then the Chern-Simons term (3.3) is rewritten as

$$
S_{\mathrm{CS}}=\int d^{3} x \frac{k}{2 \pi} \epsilon^{\mu \nu \rho} A_{\mu}^{+} F_{\nu \rho}^{-},
$$

where $F_{\mu \nu}^{-}=\partial_{\mu} A_{\nu}^{-}-\partial_{\nu} A_{\mu}^{-}$. The covariant derivative contains only $A_{\mu}^{-}$,

$$
D_{\mu} Y^{A}=\partial_{\mu} Y^{A}+2 i A_{\mu}^{-} Y^{A}
$$

The $\mathrm{U}(1)$ gauge field $A_{\mu}^{+}$is an auxiliary field which does not couple to any matter fields and its equation of motion is $F_{\mu \nu}^{-}=0$. This makes the other $\mathrm{U}(1)$ gauge field $A_{\mu}^{-}$a pure gauge degree. If we are interested in the physics blind to the orbifold structure, every covariant derivative can be replaced by an ordinary partial derivative through a gauge transformation and consequently both $\mathrm{U}(1)$ gauge fields completely decouple from the action (2.3).

Finally, we see that in (2.3) the transverse scalar fields $Y^{A}$ are complex, while in (3.2) the transverse scalar fields $X^{I}$ are real. Similarly, the 3 -form fields $C_{(3)}$ are complex, while $\hat{C}_{(3)}$ are real. Therefore, in order to complete the matching between the actions (2.3) and (3.2) we relate the complex and real scalar fields as in (2.1), which leads to the following identifications among complex and real 3-form fields:

$$
\begin{aligned}
C_{\mu \nu \rho} & =\hat{C}_{\mu \nu \rho}, \quad C_{\mu \nu A}=\frac{1}{2}\left(\hat{C}_{\mu \nu A}-i \hat{C}_{\mu \nu A+4}\right) \\
C_{\mu A B} & =\frac{1}{4}\left(\hat{C}_{\mu A B}-i \hat{C}_{\mu A+4 B}-i \hat{C}_{\mu A B+4}-\hat{C}_{\mu A+4 B+4}\right) \\
C_{\mu A \bar{B}} & =\frac{1}{4}\left(\hat{C}_{\mu A B}-i \hat{C}_{\mu A+4 B}+i \hat{C}_{\mu A B+4}+\hat{C}_{\mu A+4 B+4}\right), \\
C_{A B C} & =\frac{1}{8}\left(\hat{C}_{A B C}-i \hat{C}_{A+4 B C}-i \hat{C}_{A B+4 C}-i \hat{C}_{A B C+4}\right. \\
& \left.-\hat{C}_{A B+4 C+4}-\hat{C}_{A+4 B C+4}-\hat{C}_{A+4 B+4 C}+i \hat{C}_{A+4 B+4 C+4}\right), \\
C_{A B \bar{C}} & =\frac{1}{8}\left(\hat{C}_{A B C}-i \hat{C}_{A+4 B C}-i \hat{C}_{A B+4 C}+i \hat{C}_{A B C+4}\right. \\
& \left.+\hat{C}_{A B+4 C+4}+\hat{C}_{A+4 B C+4}-\hat{C}_{A+4 B+4 C}-i \hat{C}_{A+4 B+4 C+4}\right),
\end{aligned}
$$

and their complex conjugates. Through these identifications, the action in (2.3) exactly coincides with (3.2). This simple case supports the validity of the proposed actions in (2.3) and (2.8), however we need further check for the general $\mathrm{U}(N) \times \mathrm{U}(N)$ case in the subsequent sections.

Note that our result contains that of [12] as a particular case, where only $C_{\mu A \bar{B}}$ and its complex conjugate are taken into account. Since those specific components do not carry orbifold charge (the charge with respect to $A_{\mu}^{-}$), they could be regarded as functions on the orbifold. In fact $C_{(3)}$ is a differential form, and then each component of it should be regarded as a section on the orbifold rather than a function. This allows us to take into account all components of $C_{(3)}$ even after orbifolding. 


\section{Reduction from M-theory to IIA String Theory}

In section 2 we constructed the WZ-type action, (2.3) and (2.8), describing the couplings between the world-volume fields on multiple M2-branes and the bulk form fields. Our proposal for these couplings is guided by gauge invariance and analogy with the coupling of D-branes to the R-R form fields in type II string theories [5, 6, 7]. However, for the effective theory of multiple M2-branes there is no known guiding principle on the general form of coupling between the world-volume fields and the bulk form fields. On the other hand, in type II string theories, the coupling between the R-R form fields and the world-volume fields of D-branes is restricted by consistency with T-duality. Therefore, in order to test the consistency of our proposal, we will compactify our WZtype action in (2.3) and (2.8) and then we will compare with the corresponding WZ-type action in type IIA string theory. We first study the simplest case of ABJM theory with $\mathrm{U}(1) \times \mathrm{U}(1)$ gauge symmetry, and then generalize to the case of $\mathrm{U}(N) \times \mathrm{U}(N)$ gauge symmetry.

\section{$4.1 \mathrm{U}(1) \times \mathrm{U}(1)$ gauge symmetry}

In type IIA string theory the bosonic part of the effective action for a single $\mathrm{D} p$-brane i.2

$$
S_{10}=S_{\mathrm{DBI}}+S_{\tilde{C}}
$$

$S_{\text {DBI }}$ is the Abelian DBI action and $S_{\tilde{C}}$ is the Abelian WZ-type coupling, which is given by [7]

$$
S_{\tilde{C}}=\mu_{p} \int_{p+1}\left(P\left[\sum \tilde{C}_{(n)} e^{\tilde{B}}\right] e^{\tilde{\lambda} \tilde{F}}\right),
$$

where $\mu_{p}$ is the $\mathrm{R}$ - $\mathrm{R}$ charge of the $\mathrm{D} p$-brane, $\tilde{\lambda}=2 \pi l_{\mathrm{s}}^{2}$ is the string scale, $\tilde{B}$ is the NS-NS 2 -form field, $\tilde{F}=d \tilde{A}$ is field strength of the $\mathrm{U}(1)$ gauge field $\tilde{A}$ of the D-brane, and $\mathrm{P}[\ldots]$ is the pull-back on to the D-brane world-volume. For later convenience we expand this action explicitly for $p=2$ and $\tilde{C}_{(3)}$, omitting couplings to other fields,

$$
\begin{gathered}
S_{\tilde{C}}=\mu_{2} \int \frac{1}{3 !} d^{3} x \epsilon^{\mu \nu \rho}\left(\tilde{C}_{\mu \nu \rho}+3 \tilde{\lambda} \tilde{C}_{\mu \nu i} \tilde{D}_{\rho} \tilde{X}^{i}+3 \tilde{\lambda}^{2} \tilde{C}_{\mu i j} \tilde{D}_{\nu} \tilde{X}^{i} \tilde{D}_{\rho} \tilde{X}^{j}\right. \\
\left.+\tilde{\lambda}^{3} \tilde{C}_{i j k} \tilde{D}_{\mu} \tilde{X}^{i} \tilde{D}_{\nu} \tilde{X}^{j} \tilde{D}_{\rho} \tilde{X}^{k}+\ldots\right)
\end{gathered}
$$

where $\tilde{X}^{i}(i=1,2, \ldots, 7)$ are seven transverse scalar fields with $\tilde{D}_{\mu} \tilde{X}^{i}=\partial_{\mu} \tilde{X}^{i}$.

We note that in the $\mathrm{U}(1) \times \mathrm{U}(1)$ case the scalar fields $Y^{A}$ commute with each other. As a result the three commutator $\beta_{C}^{A B}$ is vanishing and hence the couplings to the 6 -form field are zero. In addition, the 3 -form field should be antisymmetric in all its global indices. To compactify one

\footnotetext{
${ }^{2}$ We put tildes for the fields and the parameters in string theory.
} 
direction transverse to the M2-brane, we apply the MP Higgsing procedure [13] to (2.3). To that end we turn on a vacuum expectation value (vev) for the complex scalar $Y^{4}$ as

$$
Y^{4}=\frac{v}{2}+\tilde{X}^{4}+i \tilde{X}^{8}
$$

while the remaining 3 complex scalars have vanishing vev,

$$
Y^{a}=\tilde{X}^{a}+i \tilde{X}^{a+4}, \quad(a=1,2,3) .
$$

Here the 8 real scalar fields, $\tilde{X}^{I}=X^{I}(I=1, \ldots, 8)$, stand for 8 transverse directions. The nonvanishing vev breaks the $\mathrm{U}(1) \times \mathrm{U}(1)$ gauge symmetry to the diagonal $\mathrm{U}(1)$. Then we take the double scaling limit of infinite vev $v$ and the Chern-Simons level $k$, keeping $v / k$ finite. In this limit the gauge field $A_{\mu}^{-}$becomes nondynamical while $A_{\mu}^{+}$will be a dynamical gauge field. Using (3.4), the covariant derivatives of the transverse scalar fields (2.4) can be written to the leading order in $v$ as

$$
D_{\mu} Y^{4}=\partial_{\mu} \tilde{X}^{4}+i v A_{\mu}^{-}, \quad D_{\mu} Y^{a}=\partial_{\mu} \tilde{X}^{a}+i \partial_{\mu} \tilde{X}^{a+4},
$$

where we have chosen unitary gauge for the field $A_{\mu}^{-}, A_{\mu}^{-}-\frac{1}{v} \partial_{\mu} \tilde{X}^{8} \rightarrow A_{\mu}^{-}$.

Next we need to relate the 10-dimensional real 3-form fields with the 11-dimensional complex 3 -form fields. For the first term in (2.3) this relation is obvious and is given by

$$
\tilde{C}_{\mu \nu \rho}=C_{\mu \nu \rho}+C_{\mu \nu \rho}^{\dagger} .
$$

To find the relations for the remaining terms we plug (4.6) into the action (2.3). Then from the second term in (2.3) we have

$$
\begin{aligned}
C_{\mu \nu A} D_{\rho} Y^{A}+\text { c.c. }= & \left(C_{\mu \nu 4}+C_{\mu \nu 4}^{\dagger}\right) \partial_{\rho} \tilde{X}^{4}+i v\left(C_{\mu \nu 4}-C_{\mu \nu 4}^{\dagger}\right) A_{\rho}^{-} \\
& +\left(C_{\mu \nu a}+C_{\mu \nu a}^{\dagger}\right) \partial_{\rho} \tilde{X}^{a}+i\left(C_{\mu \nu a}-C_{\mu \nu a}^{\dagger}\right) \partial_{\rho} \tilde{X}^{a+4} \\
= & \tilde{C}_{\mu \nu i} \partial_{\rho} \tilde{X}^{i}+v \tilde{B}_{\mu \nu} A_{\rho}^{-},
\end{aligned}
$$

where we have made the following identification of the R-R 3-form field and the NS-NS 2-form field in 10-dimensions,

$$
\begin{aligned}
& \tilde{C}_{\mu \nu a}=\left(C_{\mu \nu a}+C_{\mu \nu a}^{\dagger}\right), \quad \tilde{C}_{\mu \nu 4}=\left(C_{\mu \nu 4}+C_{\mu \nu 4}^{\dagger}\right), \\
& \tilde{C}_{\mu \nu a+4}=i\left(C_{\mu \nu a}-C_{\mu \nu a}^{\dagger}\right), \quad \tilde{B}_{\mu \nu}=i\left(C_{\mu \nu 4}-C_{\mu \nu \overline{4}}\right) .
\end{aligned}
$$


Similarly, from the third and fourth terms of the action (2.3) we get

$$
\begin{aligned}
\epsilon^{\mu \nu \rho}( & \left.C_{\mu A B} D_{\nu} Y^{A} D_{\rho} Y^{B}+C_{\mu A \bar{B}} D_{\nu} Y^{A} D_{\rho} Y_{B}^{\dagger}+\text { c.c. }\right) \\
=\epsilon^{\mu \nu \rho} & \left(\left(-C_{\mu 4 \bar{a}}-C_{\mu 4 \bar{a}}^{\dagger}+2 C_{\mu a 4}+2 C_{\mu a 4}^{\dagger}+C_{\mu a \overline{4}}+C_{\mu a \overline{4}}^{\dagger}\right) \partial_{\nu} \tilde{X}^{a} \partial_{\rho} \tilde{X}^{4}\right. \\
& +i\left(-C_{\mu 4 \bar{a}}+C_{\mu 4 \bar{a}}^{\dagger}+2 C_{\mu a 4}-2 C_{\mu a 4}^{\dagger}+C_{\mu a \overline{4}}-C_{\mu a \overline{4}}^{\dagger}\right) \partial_{\nu} \tilde{X}^{4} \partial_{\rho} \tilde{X}^{a+4} \\
& +i v\left(C_{\mu 4 \bar{a}}-C_{\mu 4 \bar{a}}^{\dagger}-2 C_{\mu a 4}+2 C_{\mu a 4}^{\dagger}+C_{\mu a \overline{4}}-C_{\mu a \overline{4}}^{\dagger}\right) A_{\nu}^{-} \partial_{\rho} \tilde{X}^{a} \\
& +v\left(C_{\mu 4 \bar{a}}+C_{\mu 4 \bar{a}}^{\dagger}+2 C_{\mu a 4}+2 C_{\mu a 4}^{\dagger}-C_{\mu a \overline{4}}-C_{\mu a \overline{4}}^{\dagger}\right) A_{\nu}^{-} \partial_{\rho} \tilde{X}^{a+4} \\
& +2 i v\left(C_{\mu 4 \overline{4}}-C_{\mu 4 \overline{4}}^{\dagger}\right) A_{\nu}^{-} \partial_{\rho} \tilde{X}^{4}+\left(C_{\mu a b}+C_{\mu a b}^{\dagger}+C_{\mu a \bar{b}}+C_{\mu a \bar{b}}^{\dagger}\right) \partial_{\nu} \tilde{X}^{a} \partial_{\rho} \tilde{X}^{b} \\
& +i\left(2 C_{\mu a b}-2 C_{\mu a b}^{\dagger}-C_{\mu a \bar{b}}+C_{\mu a \bar{b}}^{\dagger}-C_{\mu b \bar{a}}+C_{\mu b \bar{a}}^{\dagger}\right) \partial_{\nu} \tilde{X}^{a} \partial_{\rho} \tilde{X}^{b+4} \\
& \left.+\left(-C_{\mu a b}-C_{\mu a b}^{\dagger}+C_{\mu a \bar{b}}+C_{\mu a \bar{b}}^{\dagger}\right) \partial_{\nu} \tilde{X}^{a+4} \partial_{\rho} \tilde{X}^{b+4}\right) \\
=\epsilon^{\mu \nu \rho} & \left(\tilde{C}_{\mu i j} \partial_{\nu} \tilde{X}^{i} \partial_{\rho} \tilde{X}^{j}+v \tilde{B}_{\mu i} A_{\nu}^{-} \partial_{\rho} \tilde{X}^{i}\right) .
\end{aligned}
$$

The last step means we have made the identification of $\tilde{C}_{\mu i j}$ and $\tilde{B}_{\mu i}$ as

$$
\begin{aligned}
& \tilde{B}_{\mu a}=i\left(C_{\mu 4 \bar{a}}-C_{\mu 4 \bar{a}}^{\dagger}-2 C_{\mu a 4}+2 C_{\mu a 4}^{\dagger}+C_{\mu a \overline{4}}-C_{\mu a \overline{4}}^{\dagger}\right), \\
& \tilde{B}_{\mu a+4}=C_{\mu 4 \bar{a}}+C_{\mu 4 \bar{a}}^{\dagger}+2 C_{\mu a 4}+2 C_{\mu a 4}^{\dagger}-C_{\mu a \overline{4}}-C_{\mu a \overline{4}}^{\dagger}, \\
& \tilde{B}_{\mu 4}=2 i\left(C_{\mu 4 \overline{4}}-C_{\mu 4 \overline{4}}^{\dagger}\right), \quad \tilde{C}_{\mu a b}=C_{\mu a b}+C_{\mu a b}^{\dagger}+C_{\mu a \bar{b}}+C_{\mu a \bar{b}}^{\dagger}, \\
& \tilde{C}_{\mu a 4}=\frac{1}{2}\left(-C_{\mu 4 \bar{a}}-C_{\mu 4 \bar{a}}^{\dagger}+2 C_{\mu a 4}+2 C_{\mu a 4}^{\dagger}+C_{\mu a \overline{4}}+C_{\mu a \overline{4}}^{\dagger}\right), \\
& \tilde{C}_{\mu 4 a+4}=\frac{i}{2}\left(-C_{\mu 4 \bar{a}}+C_{\mu 4 \bar{a}}^{\dagger}+2 C_{\mu a 4}-2 C_{\mu a 4}^{\dagger}+C_{\mu a \overline{4}}-C_{\mu a \overline{4}}^{\dagger}\right), \\
& \tilde{C}_{\mu a b+4}=\frac{i}{2}\left(2 C_{\mu a b}-2 C_{\mu a b}^{\dagger}-C_{\mu a \bar{b}}+C_{\mu a \bar{b}}^{\dagger}-C_{\mu b \bar{a}}+C_{\mu b \bar{a}}^{\dagger}\right), \\
& \tilde{C}_{\mu a+4 b+4}=-C_{\mu a b}-C_{\mu a b}^{\dagger}+C_{\mu a \bar{b}}+C_{\mu a \bar{b}}^{\dagger} .
\end{aligned}
$$

For the last two terms of the action (2.3), we have

$$
\begin{aligned}
& \epsilon^{\mu \nu \rho}\left(C_{A B C} D_{\mu} Y^{A} D_{\nu} Y^{B} D_{\rho} Y^{C}+C_{A B \bar{C}} D_{\mu} Y^{A} D_{\nu} Y^{B} D_{\rho} Y_{C}^{\dagger}\right)+\text { c.c. } \\
= & \epsilon^{\mu \nu \rho}\left(\tilde{C}_{i j k} \partial_{\mu} \tilde{X}^{i} \partial_{\nu} \tilde{X}^{j} \partial_{\rho} \tilde{X}^{k}+v \tilde{B}_{i j} A_{\mu}^{-} \partial_{\nu} \tilde{X}^{i} \partial_{\rho} \tilde{X}^{j}\right) .
\end{aligned}
$$


where as usual we made the following identification of the 10-dimensional form fields

$$
\begin{aligned}
& \tilde{B}_{a 4}=-2 i\left(C_{a 4 \overline{4}}-C_{a 4 \overline{4}}^{\dagger}\right), \quad \tilde{B}_{4 a+4}=-2\left(C_{a 4 \overline{4}}+C_{a 4 \overline{4}}^{\dagger}\right), \\
& \tilde{B}_{a b}=i\left(3 C_{a b 4}-3 C_{a b 4}^{\dagger}+2 C_{a \bar{b} 4}-2 C_{a \overline{4} 4}^{\dagger}-C_{a b \overline{4}}+C_{a b \overline{4}}^{\dagger}\right), \\
& \tilde{B}_{a b+4}=-3 C_{4 a b}-3 C_{a b 4}^{\dagger}+C_{a b \overline{4}}+C_{a b \overline{4}}^{\dagger}+C_{a \bar{b} 4}+C_{a \bar{b} 4}^{\dagger}+C_{b \bar{a} 4}+C_{b \bar{a} 4}^{\dagger}, \\
& \tilde{B}_{a+4 b+4}=i\left(-3 C_{a b 4}+3 C_{a b 4}^{\dagger}+2 C_{a \bar{b} 4}-2 C_{a \bar{b} 4}^{\dagger}+C_{a b \overline{4}}-C_{a b \overline{4}}^{\dagger}\right), \\
& \tilde{C}_{a b 4}=\frac{1}{3}\left(3 C_{a b 4}+3 C_{a b 4}^{\dagger}+2 C_{a \bar{b} 4}+2 C_{a \bar{b} 4}^{\dagger}+C_{a b \overline{4}}+C_{a b \overline{4}}^{\dagger}\right), \\
& \tilde{C}_{a 4 b+4}=\frac{i}{3}\left(-3 C_{4 a b}+3 C_{a b 4}^{\dagger}-C_{a b \overline{4}}+C_{a b \overline{4}}^{\dagger}+C_{a \bar{b} 4}-C_{a \bar{b} 4}^{\dagger}+C_{b \bar{a} 4}-C_{b \bar{a} 4}^{\dagger}\right), \\
& \tilde{C}_{4 a+4 b+4}=\frac{1}{3}\left(-3 C_{a b 4}-3 C_{a b 4}^{\dagger}+2 C_{a \bar{b} 4}+2 C_{a \bar{b} 4}^{\dagger}-C_{a b \overline{4}}-C_{a b \overline{4}}^{\dagger}\right), \\
& \tilde{C}_{a b c}=\left(C_{a b c}+C_{a b c}^{\dagger}+C_{a b \bar{c}}+C_{a b \bar{c}}^{\dagger}\right), \\
& \tilde{C}_{a b c+4}=\frac{i}{3}\left(3 C_{a b c}-3 C_{a b c}^{\dagger}-C_{a b \bar{c}}+C_{a b \bar{c}}^{\dagger}-C_{a c \bar{b}}+C_{a c \bar{b}}^{\dagger}-C_{c b \bar{a}}+C_{c b \bar{a}}^{\dagger}\right), \\
& \tilde{C}_{a b+4 c+4}=\frac{1}{3}\left(-3 C_{a b c}-3 C_{a b c}^{\dagger}+2 C_{a b \bar{c}}+2 C_{a b \bar{c}}^{\dagger}+C_{c b \bar{a}}+C_{c b \bar{a}}^{\dagger}\right), \\
& \tilde{C}_{a+4 b+4 c+4}=i\left(-C_{a b c}+C_{a b c}^{\dagger}+C_{a b \bar{c}}-C_{a b \bar{c}}^{\dagger}\right) .
\end{aligned}
$$

Collecting all the terms, we obtain

$$
\begin{gathered}
S_{\tilde{C}}=\mu_{2} \int d^{3} x \frac{1}{3 !} \epsilon^{\mu \nu \rho}\left[\tilde{C}_{\mu \nu \rho}+3 \lambda\left(\tilde{C}_{\mu \nu i} \partial_{\rho} \tilde{X}^{i}+v \tilde{B}_{\mu \nu} A_{\rho}^{-}\right)+3 \lambda^{2}\left(\tilde{C}_{\mu i j} \partial_{\nu} \tilde{X}^{i} \partial_{\rho} \tilde{X}^{j}+v \tilde{B}_{\mu i} A_{\nu}^{-} \partial_{\rho} \tilde{X}^{i}\right)\right. \\
\left.+\lambda^{3}\left(\tilde{C}_{i j k} \partial_{\mu} \tilde{X}^{i} \partial_{\nu} \tilde{X}^{j} \partial_{\rho} \tilde{X}^{k}+v \tilde{B}_{i j} A_{\mu}^{-} \partial_{\nu} \tilde{X}^{i} \partial_{\rho} \tilde{X}^{j}\right)\right] .
\end{gathered}
$$

Next we integrate out the auxiliary field $A_{\mu}^{-}$. In order to do so, we should take into account the bosonic part of the original $\mathrm{U}(1) \times \mathrm{U}(1)$ ABJM action. After the MP compactification procedure, the leading order in $v$ of the ABJM action takes the following form,

$$
\int d^{3} x\left(-\tilde{D}_{\mu} \tilde{X}^{i} \tilde{D}^{\mu} \tilde{X}^{i}-v^{2} A_{\mu}^{-} A^{-\mu}+\frac{k}{2 \pi} \epsilon^{\mu \nu \rho} A_{\mu}^{-} \tilde{F}_{\nu \rho}\right)+\mathcal{O}\left(\frac{1}{v}\right),
$$

where $\tilde{F}_{\mu \nu}=\partial_{\mu} A_{\nu}^{+}-\partial_{\nu} A_{\mu}^{+}$. The equation of motion for the auxiliary field $A_{\mu}^{-}$can be obtained from the variation of (4.14) and (4.15). Solving this equation of motion to the leading order in $v$ expresses the auxiliary fields in terms of dynamical fields as

$$
A_{\mu}^{-}=\frac{k}{4 \pi v^{2}} \epsilon_{\mu}{ }^{\nu \rho}\left(\tilde{F}_{\nu \rho}+\mu_{2} v \lambda \frac{2 \pi}{k} P\left[\tilde{B}_{\nu \rho}\right]\right)=\frac{1}{2 g_{\mathrm{YM}} v} \epsilon_{\mu}^{\nu \rho}\left(\tilde{F}_{\nu \rho}+\frac{1}{\tilde{\lambda}} P\left[\tilde{B}_{\nu \rho}\right]\right) .
$$

where

$$
\begin{aligned}
& P\left[\tilde{B}_{\mu \nu}\right]=\frac{1}{2}\left(\tilde{B}_{\mu \nu}+\lambda \tilde{B}_{\mu i} \partial_{\nu} \tilde{X}^{i}+\frac{\lambda^{2}}{3} \tilde{B}_{i j} \partial_{\nu} \tilde{X}^{i} \partial_{\rho} \tilde{X}^{j}\right), \\
& g_{\mathrm{YM}}=\frac{2 \pi v}{k}, \quad g_{\mathrm{s}}=g_{\mathrm{YM}}^{2} l_{\mathrm{s}}, \quad \tilde{\lambda}=2 \pi l_{\mathrm{s}}^{2}
\end{aligned}
$$


with $\mu_{2} \lambda=1 / 2 \pi l_{\mathrm{P}}^{3 / 2}$ and $l_{\mathrm{P}}=g_{\mathrm{s}}^{1 / 3} l_{\mathrm{s}}$. For dimensional reason we also rescale the scalar fields as

$\tilde{X}^{i} \rightarrow \frac{\tilde{X}^{i}}{g_{\mathrm{YM}}}$. Substituting $A_{\mu}^{-}$into the actions (4.14) and (4.15), and rearranging the terms, we obtain

$$
\begin{aligned}
S_{10}=\int d^{3} x\left\{\frac{\mu_{2}}{3 !} \epsilon^{\mu \nu \rho}[\right. & \left.\tilde{C}_{\mu \nu \rho}+3 \tilde{\lambda} \tilde{C}_{\mu \nu i} \partial_{\rho} \tilde{X}^{i}+3 \tilde{\lambda}^{2} \tilde{C}_{\mu i j} \partial_{\nu} \tilde{X}^{i} \partial_{\rho} \tilde{X}^{j}+\tilde{\lambda}^{3} \tilde{C}_{i j k} \partial_{\mu} \tilde{X}^{i} \partial_{\nu} \tilde{X}^{j} \partial_{\rho} \tilde{X}^{k}\right] \\
& \left.+\frac{1}{g_{\mathrm{YM}}^{2}}\left[-\tilde{D}_{\mu} \tilde{X}^{i} \tilde{D}^{\mu} \tilde{X}^{i}-\frac{1}{2}\left(\tilde{F}_{\mu \nu}+\frac{1}{\tilde{\lambda}} P\left[\tilde{B}_{\mu \nu}\right]\right)^{2}\right]\right\} .
\end{aligned}
$$

As anticipated, the reduction to 10-dimensions results in the linearized DBI action and the coupling of the R-R 3-form field to D2-brane (4.3).

\section{$4.2 \quad \mathrm{U}(N) \times \mathbf{U}(N)$ gauge symmetry}

In the pervious section we have verified that, when compactified to 10 -dimensions, the $\mathrm{U}(1) \times \mathrm{U}(1)$ ABJM theory coupled to the 11-dimensional 3-form field gives rise to the theory of a single D2brane coupled to the 10-dimensional R-R 3-form field and the NS-NS 2-form field. In this section we will extend this procedure to the case of non-Abelian gauge symmetry. In particular we will consider the $\mathrm{U}(N) \times \mathrm{U}(N)$ ABJM theory coupled to the 11-dimensional 3-form and 6-form fields. In string theory, the non-Abelian extension of (4.2) is given in [7] as

$$
S_{\tilde{C}}=\mu_{p} \int_{p+1} \operatorname{Tr}\left(P\left[e^{i \tilde{i} \tilde{X}^{2}} \sum \tilde{C}_{(n)} e^{\tilde{B}}\right] e^{\tilde{\lambda} \tilde{F}}\right)
$$

where $\mathbf{i}_{\tilde{X}}$ denotes the contraction (interior product) with $\tilde{X}^{i}$. Note that since $\tilde{X}^{i}$ is now the $N \times N$ matrix, $\mathbf{i}_{\tilde{X}}^{2}$ is nonzero and given in terms of the commutator of $\tilde{X}^{i}$.

For clarity of presentation we treat the terms involving the 3- and 6-form fields separately. The reduction to 10-dimension is achieved by breaking the $\mathrm{U}(N) \times \mathrm{U}(N)$ gauge symmetry down to $\mathrm{U}(N)$, and the scalar fields are in the adjoint representation of the unbroken $\mathrm{U}(N)$. Therefore, the transverse scalars $X^{I}$ introduced in (2.1) can be split into its trace and traceless part as

$$
X^{I}=\check{X}^{I}+i \hat{X}^{I}=\check{X}_{0}^{I} T^{0}+i \hat{X}_{\alpha}^{I} T^{\alpha}
$$

where $T^{0}$ and $T^{\alpha}\left(\alpha=1, \ldots, N^{2}-1\right)$ are the generators of the unbroken $\mathrm{U}(N)$. Then the covariant derivative (2.4) becomes

$$
D_{\mu} Y^{A}=\tilde{D}_{\mu} X^{A}+i \tilde{D}_{\mu} X^{A+4}+i\left\{A_{\mu}^{-}, X^{A}+i X^{A+4}\right\}
$$

where we used non-Abelian version of (3.4) and $\tilde{D}_{\mu} X=\partial_{\mu} X+i\left[A_{\mu}^{+}, X\right]$. 
Now let us turn on vev for the trace part of $Y^{4}$ as

$$
Y^{4}=\frac{v}{2} T^{0}+X^{4}+i X^{8}
$$

and introduce 7 Hermitian scalars fields in the adjoint representation of the $\mathrm{U}(N)$ :

$$
\tilde{X}^{4}=\check{X}^{4}-\hat{X}^{8}, \quad \tilde{X}^{a}=\check{X}^{a}-\hat{X}^{a+4}, \quad \tilde{X}^{a+4}=\check{X}^{a+4}+\hat{X}^{a}, \quad(a=1,2,3) .
$$

In the double scaling limit of infinite vev $v$ and the Chern-Simons level $k$, the covariant derivative (4.21) to the leading order in $v$ becomes

$$
\begin{aligned}
& D_{\mu} Y^{4}=\tilde{D}_{\mu}\left(\check{X}^{4}-\hat{X}^{8}\right)+i v\left[A_{\mu}^{-}+\frac{1}{v}\left(\tilde{D}_{\mu}\left(\check{X}^{8}+\hat{X}^{4}\right)\right)\right]=\tilde{D}_{\mu} \tilde{X}^{4}+i v A_{\mu}^{-}, \\
& D_{\mu} Y^{a}=\tilde{D}_{\mu}\left[\left(\check{X}^{a}+i \hat{X}^{a}\right)+i\left(\check{X}^{a+4}+i \hat{X}^{a+4}\right)\right]=\tilde{D}_{\mu} \tilde{X}^{a}+i \tilde{D}_{\mu} \tilde{X}^{a+4}
\end{aligned}
$$

where we have made a gauge choice where $A_{\mu}^{-} \rightarrow A_{\mu}^{-}-\frac{1}{v} \tilde{D}_{\mu}\left(\check{X}^{8}+\hat{X}^{4}\right)$. In the same limit, the three commutator terms $\beta_{C}^{A B}$ are reduced to

$$
\begin{aligned}
\beta_{4}^{a 4} & =\frac{v}{2}\left(\left[\tilde{X}^{a}, \tilde{X}^{4}\right]+i\left[\tilde{X}^{a+4}, \tilde{X}^{4}\right]\right), \\
\beta_{4}^{a b} & =\frac{v}{4}\left(\left[\tilde{X}^{a}, \tilde{X}^{b}\right]+i\left[\tilde{X}^{a}, \tilde{X}^{b+4}\right]+i\left[\tilde{X}^{a+4}, \tilde{X}^{b}\right]-\left[\tilde{X}^{a+4}, \tilde{X}^{b+4}\right]\right), \\
\beta_{b}^{a 4} & =\frac{v}{4}\left(\left[\tilde{X}^{a}, \tilde{X}^{b}\right]-i\left[\tilde{X}^{a}, \tilde{X}^{b+4}\right]+i\left[\tilde{X}^{a+4}, \tilde{X}^{b}\right]+\left[\tilde{X}^{a+4}, \tilde{X}^{b+4}\right]\right) .
\end{aligned}
$$

\subsubsection{3-form fields}

As has been done in the previous subsection, we calculate each term in the WZ-type action (2.3) in the compactification limit, and identify it with the corresponding WZ-type coupling in type IIA string theory. The first term in (2.3) can simply be identified with the corresponding 3-form field in type IIA string theory as

$$
\tilde{C}_{\mu \nu \rho}=C_{\mu \nu \rho}+C_{\mu \nu \rho}^{\dagger}
$$

Using (4.24), from the second term in (2.3) we obtain

$$
C_{\mu \nu A} D_{\rho} Y^{A}+\text { c.c. }=\tilde{C}_{\mu \nu i} D_{\rho} \tilde{X}^{i}+v \tilde{B}_{\mu \nu} A_{\rho}^{-}
$$

where we have identified the 3-form fields in M-theory with the R-R and NS-NS form fields in IIA string theory as

$$
\begin{aligned}
\tilde{C}_{\mu \nu a+4} & =i\left(C_{\mu \nu a}-C_{\mu \nu a}^{\dagger}\right), & \tilde{C}_{\mu \nu a} & =C_{\mu \nu a}+C_{\mu \nu a}^{\dagger}, \\
\tilde{C}_{\mu \nu 4} & =C_{\mu \nu 4}+C_{\mu \nu 4}^{\dagger}, & \tilde{B}_{\mu \nu} & =i\left(C_{\mu \nu 4}-C_{\mu \nu 4}^{\dagger}\right) .
\end{aligned}
$$


For the remaining terms in (2.3) the reduction to 10-dimensions seems more subtle. As an example let us consider the following particular term:

$$
\{\operatorname{Tr}\}\left(C_{\mu A \bar{B}} D_{\nu} Y^{A} D_{\rho} Y_{B}^{\dagger}\right)=\left(C_{\mu A \bar{B}}\right)_{a \hat{b}}^{\hat{a} b}\left(D_{\nu} Y^{A}\right)_{\hat{a}}^{a}\left(D_{\rho} Y_{B}^{\dagger}\right)_{b}^{\hat{b}}
$$

After the symmetry breaking the complex scalar fields and their complex conjugates are all in the adjoint representation of the unbroken $\mathrm{U}(N)$. Therefore, the hatted and unhatted gauge indices are indistinguishable so that we may write

$$
\{\operatorname{Tr}\}\left(C_{\mu A \bar{B}} D_{\nu} Y^{A} D_{\rho} Y_{B}^{\dagger}\right)=\left(C_{\mu A \bar{B}}\right)_{a b}^{c d}\left(D_{\nu} Y^{A}\right)_{c}^{a}\left(D_{\rho} Y_{B}^{\dagger}\right)_{d}^{b}
$$

Based on the Taylor expansion in (2.7), in the compactification limit, the leading order terms of $\left(C_{\mu A \bar{B}}\right)_{a b}^{c d}$ are given by

$$
\left(C_{\mu A \bar{B}}\right)_{a b}^{c d}=\delta_{a}^{d}\left(C_{\mu A \bar{B}}^{(1)}\right)_{b}^{c}+\delta_{b}^{c}\left(C_{\mu A \bar{B}}^{(2)}\right)_{a}^{d}
$$

where $C^{(1)}$ and $C^{(2)}$ can depend on worldvolume coordinates as well as transverse scalar fields 3 As a result the WZ-type coupling in (4.30) gives

$$
\{\operatorname{Tr}\}\left(C_{\mu A \bar{B}} D_{\nu} Y^{A} D_{\rho} Y_{B}^{\dagger}\right)=\operatorname{Tr}\left(C_{\mu A \bar{B}}^{(1)} D_{\nu} Y^{A} D_{\rho} Y_{B}^{\dagger}\right)+\operatorname{Tr}\left(C_{\mu A \bar{B}}^{(2)} D_{\rho} Y_{B}^{\dagger} D_{\nu} Y^{A}\right),
$$

where ' $\mathrm{Tr}$ ' on the right-hand side represents the ordinary trace of $N \times N$ matrices. From the WZ-type coupling in (4.30), we get the two types of terms in (4.32) and have introduced two types of form fields, $C_{\mu A \bar{B}}^{(1)}$ and $C_{\mu A \bar{B}}^{(2)}$. This is true for the other terms involving both $D_{\mu} Y^{A}$ and $D_{\mu} Y_{A}^{\dagger}$. However, for the terms involving only $D_{\mu} Y^{A}$ or only $D_{\mu} Y_{A}^{\dagger}$, we have only one type of term. For instance

$$
\begin{aligned}
\epsilon^{\mu \nu \rho}\{\operatorname{Tr}\}\left(C_{\mu A B} D_{\nu} Y^{A} D_{\rho} Y^{B}\right) & =\epsilon^{\mu \nu \rho} \operatorname{Tr}\left(\hat{C}_{\mu A B}^{(1)} D_{\nu} Y^{A} D_{\rho} Y^{B}+\hat{C}_{\mu A B}^{(2)} D_{\rho} Y^{B} D_{\nu} Y^{A}\right) \\
& =\epsilon^{\mu \nu \rho} \operatorname{Tr}\left(C_{\mu A B}^{(3)} D_{\nu} Y^{A} D_{\rho} Y^{B}\right),
\end{aligned}
$$

where $C_{\mu A B}^{(3)}=\hat{C}_{\mu A B}^{(1)}-\hat{C}_{\mu B A}^{(2)}$.

Using this procedure, from the third and the fourth terms in (2.3) we have

$$
\begin{aligned}
\epsilon^{\mu \nu \rho}\{\operatorname{Tr}\}( & \left.C_{\mu A \bar{B}} D_{\nu} Y^{A} D_{\rho} Y_{B}^{\dagger}+C_{\mu A B} D_{\nu} Y^{A} D_{\rho} Y^{B}\right)+ \text { (c.c.) } \\
=\epsilon^{\mu \nu \rho} & \operatorname{Tr}\left(C_{\mu A \bar{B}}^{(1)} D_{\nu} Y^{A} D_{\rho} Y_{B}^{\dagger}-C_{\mu A \bar{B}}^{(2)} D_{\nu} Y_{B}^{\dagger} D_{\rho} Y^{A}+C_{\mu A B}^{(3)} D_{\nu} Y^{A} D_{\rho} Y^{B}\right)+(\text { c.c. }) \\
=\epsilon^{\mu \nu \rho} & \operatorname{Tr}\left(C_{\mu 4 \overline{4}}^{(1)} D_{\nu} Y^{4} D_{\rho} Y_{4}^{\dagger}-C_{\mu 4 \overline{4}}^{(2)} D_{\nu} Y_{4}^{\dagger} D_{\rho} Y^{4}+C_{\mu 44}^{(3)} D_{\nu} Y^{4} D_{\rho} Y^{4}+C_{\mu 4 \bar{a}}^{(1)} D_{\nu} Y^{4} D_{\rho} Y_{a}^{\dagger}\right. \\
& +C_{\mu a \overline{4}}^{(1)} D_{\nu} Y^{a} D_{\rho} Y_{4}^{\dagger}-C_{\mu a \overline{4}}^{(2)} D_{\nu} Y_{4}^{\dagger} D_{\rho} Y^{a}-C_{\mu 4 \bar{a}}^{(2)} D_{\nu} Y_{a}^{\dagger} D_{\rho} Y^{4}+C_{\mu 4 a}^{(3)} D_{\nu} Y^{4} D_{\rho} Y^{a} \\
& \left.+C_{\mu a 4}^{(3)} D_{\nu} Y^{a} D_{\rho} Y^{4}+C_{\mu a \bar{b}}^{(1)} D_{\nu} Y^{a} D_{\rho} Y_{b}^{\dagger}-C_{\mu a \bar{b}}^{(2)} D_{\nu} Y_{b}^{\dagger} D_{\rho} Y^{a}+C_{\mu a b}^{(3)} D_{\nu} Y^{a} D_{\rho} Y^{b}\right)+ \text { (c.c.). }
\end{aligned}
$$

\footnotetext{
${ }^{3}$ See Appendix $\mathrm{A}$ for clarifications.
} 
Then we use (4.24) to replace the covariant derivatives in (4.34). Unlike (4.27), this replacement does not exactly produce the expected WZ-type coupling in type IIA string theory. In general, when we plug (4.24) into (2.3), in addition to the expected 10-dimensional WZ-type couplings, it generates some unknown terms in string theory, involving quadratic or higher order in either $A_{\mu}^{-}$or $\tilde{D}_{\mu} \tilde{X}^{4}$. To reproduce all the known correct couplings from (4.34), we have to impose some constraints on the different types of 3 -form fields which we have introduced. These constraints are obtained by requiring that the NS-NS and R-R form fields obtained from the reduction to 10dimensions should be antisymmetric and the coefficients of the unknown terms involving $A_{\mu}^{-} A_{\nu}^{-}$ and $\tilde{D}_{\mu} \tilde{X}^{4} \tilde{D}_{\nu} \tilde{X}^{4}$ should vanish. In order to get a symmetrized 10-dimensional products we also require that the coefficients of $A_{\mu}^{-} \tilde{D}_{\nu} \tilde{X}^{i}$ and $\tilde{D}_{\nu} \tilde{X}^{i} A_{\mu}^{-}$are equal. From these conditions we obtain the following constraints:

$$
C_{\mu A \bar{B}}^{(1)}-C_{\mu B \bar{A}}^{(1) \dagger}=C_{\mu A \bar{B}}^{(2)}-C_{\mu B \bar{A}}^{(2) \dagger}, \quad C_{\mu A B}^{(3)}=C_{\mu[A B]}^{(3)}, \quad(A, B=1,2,3,4),
$$

where the notation $[A B \ldots]$ denotes the antisymmetrization of indices. With these constraints (4.34) is reduced to

$$
\begin{gathered}
\epsilon^{\mu \nu \rho}\{\operatorname{Tr}\}\left(C_{\mu A \bar{B}} D_{\nu} Y^{A} D_{\rho} Y_{B}^{\dagger}+C_{\mu A B} D_{\nu} Y^{A} D_{\rho} Y^{B}\right)+(\text { c.c. }) \\
=\epsilon^{\mu \nu \rho} \operatorname{Tr}\left(\tilde{C}_{\mu i j} \tilde{D}_{\nu} \tilde{X}^{i} \tilde{D}_{\rho} \tilde{X}^{j}+v \tilde{B}_{\mu i}\left\langle\left\langle A_{\nu}^{-} \tilde{D}_{\rho} \tilde{X}^{i}\right\rangle\right\rangle\right)
\end{gathered}
$$

where the R-R form fields $\tilde{C}_{\mu i j}$ and the NS-NS form fields $\tilde{B}_{\mu i}$ are identified as

$$
\begin{aligned}
& \tilde{B}_{\mu 4}=4 i\left(C_{\mu 4 \overline{4}}^{(1)}-C_{\mu 4 \overline{4}}^{(1) \dagger}\right), \\
& \tilde{B}_{\mu a}=2 i\left(C_{\mu 4 \bar{a}}^{(1)}-C_{\mu 4 \bar{a}}^{(1) \dagger}+C_{\mu a \overline{4}}^{(1)}-C_{\mu a \overline{4}}^{(1) \dagger}+C_{\mu 4 a}^{(3)}-C_{\mu 4 a}^{(3) \dagger}\right), \\
& \tilde{B}_{\mu a+4}=2\left(C_{\mu 4 \bar{a}}^{(1)}+C_{\mu 4 \bar{a}}^{(1) \dagger}-C_{\mu a \overline{4}}^{(1)}-C_{\mu \bar{a}}^{(1) \dagger}-C_{\mu 4 a}^{(3)}-C_{\mu 4 a}^{(3) \dagger}\right), \\
& \tilde{C}_{\mu 4 a}=C_{\mu 4 \bar{a}}^{(1)}+C_{\mu 4 \bar{a}}^{(1) \dagger}-C_{\mu a \overline{4}}^{(1)}-C_{\mu a \overline{4}}^{(1) \dagger}+C_{\mu 4 a}^{(3)}+C_{\mu 4 a}^{(3) \dagger}, \\
& \tilde{C}_{\mu 4 a+4}=-i\left(C_{\mu 4 \bar{a}}^{(1)}-C_{\mu 4 \bar{a}}^{(1) \dagger}+C_{\mu a \overline{4}}^{(1)}-C_{\mu a \overline{4}}^{(1) \dagger}-C_{\mu 4 a}^{(3)}+C_{\mu 4 a}^{(3) \dagger}\right), \\
& \tilde{C}_{\mu a b}=C_{\mu a \bar{b}}^{(1) \dagger}-C_{\mu b \bar{a}}^{(1)}+C_{\mu a \bar{b}}^{(2)}-C_{\mu b \bar{a}}^{(2) \dagger}-C_{\mu a b}^{(3) \dagger}+C_{\mu a b}^{(3)}, \\
& \tilde{C}_{\mu a b+4}=i\left(C_{\mu a \bar{b}}^{(1) \dagger}-C_{\mu b \bar{a}}^{(1)}-C_{\mu a \bar{b}}^{(2)}+C_{b \bar{a}}^{(2) \dagger}+C_{\mu a b}^{(3) \dagger}-C_{\mu b a}^{(3)}\right), \\
& \tilde{C}_{\mu a+4 b+4}=C_{\mu a \bar{b}}^{(1) \dagger}-C_{\mu b \bar{a}}^{(1)}+C_{\mu a \bar{b}}^{(2)}-C_{\mu b \bar{a}}^{(2) \dagger}+C_{\mu a b}^{(3) \dagger}-C_{\mu a b}^{(3)} .
\end{aligned}
$$

For simplicity we used a short hand notation $\langle\langle\ldots\rangle\rangle$ for symmetrized product in (4.36). For instance,

$$
\left\langle\left\langle A_{\nu}^{-} \tilde{D}_{\rho} \tilde{X}^{i}\right\rangle\right\rangle=\frac{1}{2}\left(A_{\nu}^{-} \tilde{D}_{\rho} \tilde{X}^{i}+\tilde{D}_{\rho} \tilde{X}^{i} A_{\nu}^{-}\right) .
$$


Next we consider the last two terms in (2.3). After the breaking of the gauge symmetry these terms can be written as

$$
\begin{aligned}
& \left.\epsilon^{\mu \nu \rho}\{\operatorname{Tr}\}\left(C_{A B C} D_{\mu} Y^{A} D_{\nu} Y^{B} D_{\rho} Y^{C}+C_{A B \bar{C}} D_{\mu} Y^{A} D_{\nu} Y^{B} D_{\rho} Y_{C}^{\dagger}\right)+\text { (c.c. }\right) \\
& =\epsilon^{\mu \nu \rho} \operatorname{Tr}\left(C_{A B C}^{(1)} D_{\mu} Y^{A} D_{\nu} Y^{B} D_{\rho} Y^{C}+C_{A B \bar{C}}^{(2)} D_{\mu} Y^{A} D_{\nu} Y^{B} D_{\rho} Y_{C}^{\dagger}-C_{A C \bar{B}}^{(3)} D_{\mu} Y^{A} D_{\nu} Y_{B}^{\dagger} D_{\rho} Y^{C}\right. \\
& \left.+C_{B C \bar{A}}^{(4)} D_{\mu} Y_{A}^{\dagger} D_{\nu} Y^{B} D_{\rho} Y^{C}\right)+(\text { c.c. }) \text {. } \\
& =\epsilon^{\mu \nu \rho} \operatorname{Tr}\left(C_{44 a}^{(1)} D_{\mu} Y^{4} D_{\nu} Y^{4} D_{\rho} Y^{a}+C_{4 a 4}^{(1)} D_{\mu} Y^{4} D_{\nu} Y^{a} D_{\rho} Y^{4}+C_{a 44}^{(1)} D_{\mu} Y^{a} D_{\nu} Y^{4} D_{\rho} Y^{4}\right. \\
& +C_{44 \bar{a}}^{(2)} D_{\mu} Y^{4} D_{\nu} Y^{4} D_{\rho} Y_{a}^{\dagger}+C_{4 a \overline{4}}^{(2)} D_{\mu} Y^{4} D_{\nu} Y^{a} D_{\rho} Y_{4}^{\dagger}+C_{a 4 \overline{4}}^{(2)} D_{\mu} Y^{a} D_{\nu} Y^{4} D_{\rho} Y_{4}^{\dagger} \\
& -C_{4 a \overline{4}}^{(3)} D_{\mu} Y^{4} D_{\nu} Y_{4}^{\dagger} D_{\rho} Y^{a}-C_{44 \bar{a}}^{(3)} D_{\mu} Y^{4} D_{\nu} Y_{a}^{\dagger} D_{\rho} Y^{4}-C_{a 4 \overline{4}}^{(3)} D_{\mu} Y^{a} D_{\nu} Y_{4}^{\dagger} D_{\rho} Y^{4} \\
& +C_{4 a \overline{4}}^{(4)} D_{\mu} Y_{4}^{\dagger} D_{\nu} Y^{4} D_{\rho} Y^{a}+C_{a 4 \overline{4}}^{(4)} D_{\mu} Y_{4}^{\dagger} D_{\nu} Y^{a} D_{\rho} Y^{4}+C_{44 \bar{a}}^{(4)} D_{\mu} Y_{a}^{\dagger} D_{\nu} Y^{4} D_{\rho} Y^{4} \\
& +C_{4 a b}^{(1)} D_{\mu} Y^{4} D_{\nu} Y^{a} D_{\rho} Y^{b}+C_{a 4 b}^{(1)} D_{\mu} Y^{a} D_{\nu} Y^{4} D_{\rho} Y^{b}+C_{a b 4}^{(1)} D_{\mu} Y^{a} D_{\nu} Y^{b} D_{\rho} Y^{4} \\
& +C_{4 a \bar{b}}^{(2)} D_{\mu} Y^{4} D_{\nu} Y^{a} D_{\rho} Y_{b}^{\dagger}+C_{a 4 \bar{b}}^{(2)} D_{\mu} Y^{a} D_{\nu} Y^{4} D_{\rho} Y_{b}^{\dagger}+C_{a b \overline{4}}^{(2)} D_{\mu} Y^{a} D_{\nu} Y^{b} D_{\rho} Y_{4}^{\dagger} \\
& -C_{4 b \bar{a}}^{(3)} D_{\mu} Y^{4} D_{\nu} Y_{a}^{\dagger} D_{\rho} Y^{b}-C_{a b \overline{4}}^{(3)} D_{\mu} Y^{a} D_{\nu} Y_{4}^{\dagger} D_{\rho} Y^{b}-C_{a 4 \bar{b}}^{(3)} D_{\mu} Y^{a} D_{\nu} Y_{b}^{\dagger} D_{\rho} Y^{4} \\
& +C_{a b \overline{4}}^{(4)} D_{\mu} Y_{4}^{\dagger} D_{\nu} Y^{a} D_{\rho} Y^{b}+C_{4 b \bar{a}}^{(4)} D_{\mu} Y_{a}^{\dagger} D_{\nu} Y^{4} D_{\rho} Y^{b}+C_{b 4 \bar{a}}^{(4)} D_{\mu} Y_{a}^{\dagger} D_{\nu} Y^{b} D_{\rho} Y^{4} \\
& +C_{a b c}^{(1)} D_{\mu} Y^{a} D_{\nu} Y^{b} D_{\rho} Y^{c}+C_{a b \bar{c}}^{(2)} D_{\mu} Y^{a} D_{\nu} Y^{b} D_{\rho} Y_{c}^{\dagger}-C_{a c \bar{b}}^{(3)} D_{\mu} Y^{a} D_{\nu} Y_{b}^{\dagger} D_{\rho} Y^{c} \\
& \left.+C_{b c \bar{a}}^{(4)} D_{\mu} Y_{a}^{\dagger} D_{\nu} Y^{b} D_{\rho} Y^{c}\right)+ \text { (c.c.). }
\end{aligned}
$$

In the last step we assumed the terms involving $C_{444}$ and $C_{44 \overline{4}}$ are vanishing. This is because, as we see from (4.24), those terms give rise to only terms that are quadratic or higher order in $A_{\mu}^{-}$ or $\tilde{D}_{\mu} \tilde{X}^{4}$, which are not allowed in string theory.

The next step is to replace the covariant derivatives in (4.39) by using (4.24). As we did in (4.34), we require that the R-R and NS-NS form fields which are obtained from the reduction to 10-dimensions should be antisymmetric in interchange of indices and the coefficients of the unwanted terms involving $A_{\mu}^{-} A_{\nu}^{-} \tilde{D}_{\rho} \tilde{X}^{a}$ or $\tilde{D}_{\mu} \tilde{X}^{4} \tilde{D}_{\nu} \tilde{X}^{4} \tilde{D}_{\rho} \tilde{X}^{a}$ vanish. We also impose more conditions to produce symmetrized matrix products in 10-dimensions. For instance we require that the coefficients of $\left(A_{\mu}^{-} \tilde{D}_{\nu} \tilde{X}^{a} \tilde{D}_{\rho} \tilde{X}^{b}\right),\left(\tilde{D}_{\nu} \tilde{X}^{a} A_{\mu}^{-} \tilde{D}_{\rho} \tilde{X}^{b}\right)$, and $\left(\tilde{D}_{\nu} \tilde{X}^{a} \tilde{D}_{\rho} \tilde{X}^{b} A_{\mu}^{-}\right)$are equal. The constraints from these requirements are

$$
C_{A B C}^{(1)}=C_{[A B C]}^{(1)}, \quad C_{A B \bar{C}}^{(2)}=C_{[A B] \bar{C}}^{(2)}=C_{A B \bar{C}}^{(3)}=C_{[A B] \bar{C}}^{(3)}=C_{A B \bar{C}}^{(4)}=C_{[A B] \bar{C}}^{(4)} .
$$

With these constraints we get

$$
\begin{gathered}
\epsilon^{\mu \nu \rho}\{\operatorname{Tr}\}\left(C_{A B C} D_{\mu} Y^{A} D_{\nu} Y^{B} D_{\rho} Y^{C}+C_{A B \bar{C}} D_{\mu} Y^{A} D_{\nu} Y^{B} D_{\rho} Y_{C}^{\dagger}\right)+(\text { c.c. }) \\
=\epsilon^{\mu \nu \rho} \operatorname{Tr}\left(v \tilde{B}_{i j}\left\langle\left\langle A_{\mu}^{-} \tilde{D}_{\nu} \tilde{X}^{i} \tilde{D}_{\rho} \tilde{X}^{j}\right\rangle\right\rangle+\tilde{C}_{i j k} \tilde{D}_{\mu} \tilde{X}^{i} \tilde{D}_{\nu} \tilde{X}^{j} \tilde{D}_{\rho} \tilde{X}^{k}\right),
\end{gathered}
$$


where

$$
\begin{aligned}
& \tilde{B}_{a 4}=-3 i\left(C_{a 4 \overline{4}}^{(4)}-C_{a 4 \overline{4}}^{(4) \dagger}\right), \quad \tilde{B}_{4 a+4}=-3\left(C_{a 4 \overline{4}}^{(4)}+C_{a 4 \overline{4}}^{(4) \dagger}\right), \\
& \tilde{B}_{a b}=3 i\left(C_{a b 4}^{(1)}-C_{a b 4}^{(1) \dagger}-C_{a b \overline{4}}^{(4)}+C_{a b \overline{4}}^{(4) \dagger}+C_{b 4 \bar{a}}^{(4)}-C_{b 4 \bar{a}}^{(4) \dagger}-C_{a 4 \bar{b}}^{(4)}+C_{a 4 \bar{b}}^{(4) \dagger}\right), \\
& \tilde{B}_{a b+4}=-3\left(C_{a b 4}^{(1)}+C_{a b 4}^{(1) \dagger}-C_{a b \overline{4}}^{(4)}-C_{a b \overline{4}}^{(4) \dagger}+C_{b 4 \bar{a}}^{(4)}+C_{b 4 \bar{a}}^{(4) \dagger}+C_{a 4 \bar{b}}^{(4)}+C_{a 4 \bar{b}}^{(4) \dagger}\right), \\
& \tilde{B}_{a+4 b+4}=-3 i\left(C_{a b 4}^{(1)}-C_{a b 4}^{(1) \dagger}-C_{a b \overline{4}}^{(4)}+C_{a b \overline{4}}^{(4) \dagger}-C_{b 4 \bar{a}}^{(4)}+C_{b 4 \bar{a}}^{(4) \dagger}+C_{a 4 \bar{b}}^{(4)}-C_{a 4 \bar{b}}^{(4) \dagger}\right), \\
& \tilde{C}_{a b 4}=3\left(C_{a b 4}^{(1)}+C_{a b 4}^{(1) \dagger}+C_{a b \overline{4}}^{(4)}+C_{a b \overline{4}}^{(4) \dagger}+C_{b 4 \bar{a}}^{(4)}+C_{b 4 \bar{a}}^{(4) \dagger}-C_{a 4 \bar{b}}^{(4)}-C_{a 4 \bar{b}}^{(4) \dagger}\right) \\
& \tilde{C}_{a 4 b+4}=-3\left(C_{a b 4}^{(1)}-C_{a b 4}^{(1) \dagger}+C_{a b \overline{4}}^{(4)}-C_{a b \overline{4}}^{(4) \dagger}+C_{b 4 \bar{a}}^{(4)}-C_{b 4 \bar{a}}^{(4) \dagger}+C_{a 4 \bar{b}}^{(4)}-C_{a 4 \bar{b}}^{(4) \dagger}\right), \\
& \tilde{C}_{4 a+4 b+4}=-3\left(C_{a b 4}^{(1)}+C_{a b 4}^{(1) \dagger}+C_{a b \overline{4}}^{(4)}+C_{a b \overline{4}}^{(4) \dagger}-C_{b 4 \bar{a}}^{(4)}-C_{b 4 \bar{a}}^{(4) \dagger}+C_{a 4 \bar{b}}^{(4)}+C_{a 4 \bar{b}}^{(4) \dagger}\right), \\
& \tilde{C}_{a b c}=C_{a b c}^{(1)}+C_{a b c}^{(1) \dagger}+C_{a b \bar{c}}^{(4)}+C_{a b \bar{c}}^{(4) \dagger}-C_{a c \bar{b}}^{(4)}-C_{a c \bar{b}}^{(4) \dagger}+C_{b c \bar{a}}^{(4)}+C_{b c \bar{a}}^{(4) \dagger}, \\
& \tilde{C}_{a b c+4}=i\left(C_{a b c}^{(1)}-C_{a b c}^{(1) \dagger}-C_{a b \bar{c}}^{(4)}+C_{a b \bar{c}}^{(4) \dagger}-C_{a c \bar{b}}^{(4)}+C_{a c \bar{b}}^{(4) \dagger}+C_{b c \bar{a}}^{(4)}-C_{b c \bar{a}}^{(4) \dagger}\right), \\
& \tilde{C}_{a b+4 c+4}=-\left(C_{a b c}^{(1)}+C_{a b c}^{(1) \dagger}-C_{a b \bar{c}}^{(4)}-C_{a b \bar{c}}^{(4) \dagger}+C_{a c \bar{b}}^{(4)}+C_{a c \bar{b}}^{(4) \dagger}+C_{b c \bar{a}}^{(4)}+C_{b c \bar{a}}^{(4) \dagger}\right), \\
& \tilde{C}_{a+4 b+4 c+4}=-i\left(C_{a b c}^{(1)}-C_{a b c}^{(1) \dagger}-C_{a b \bar{c}}^{(4)}+C_{a b \bar{c}}^{(4) \dagger}+C_{a c \bar{b}}^{(4)}-C_{a c \bar{b}}^{(4) \dagger}-C_{b c \bar{a}}^{(4)}+C_{b c \bar{a}}^{(4) \dagger}\right) .
\end{aligned}
$$

Inserting (4.26), (4.27), (4.36), and (4.41) into (2.3), we obtain

$$
\begin{aligned}
S_{\tilde{C}}^{(3)}=\mu_{2} & \int d^{3} x \frac{1}{3 !} \epsilon^{\mu \nu \rho}\left(\tilde{C}_{\mu \nu \rho}+3 \lambda\left(\tilde{C}_{\mu \nu i} \tilde{D}_{\rho} \tilde{X}^{i}+v \tilde{B}_{\mu \nu} A_{\rho}^{-}\right)+3 \lambda^{2}\left(\tilde{C}_{\mu i j} \tilde{D}_{\nu} \tilde{X}^{i} \tilde{D}_{\rho} \tilde{X}^{j}\right.\right. \\
& \left.\left.+v \tilde{B}_{\mu i}\left\langle\left\langle A_{\nu}^{-} \tilde{D}_{\rho} \tilde{X}^{i}\right\rangle\right\rangle\right)+\lambda^{3}\left(\tilde{C}_{i j k} \tilde{D}_{\mu} \tilde{X}^{i} \tilde{D}_{\nu} \tilde{X}^{j} \tilde{D}_{\rho} \tilde{X}^{k}+v \tilde{B}_{i j}\left\langle\left\langle A_{\mu}^{-} \tilde{D}_{\nu} \tilde{X}^{i} \tilde{D}_{\rho} \tilde{X}^{j}\right\rangle\right\rangle\right)\right) .
\end{aligned}
$$

On the other hand, after compactification the bosonic part of the original $\mathrm{U}(N) \times \mathrm{U}(N)$ ABJM action is given by

$$
\int d^{3} x \operatorname{Tr}\left(-\tilde{D}_{\mu} \tilde{X}^{i} \tilde{D}^{\mu} \tilde{X}^{i}-v^{2} A_{\mu}^{-} A^{-\mu}+\frac{k}{2 \pi} \epsilon^{\mu \nu \rho} A_{\mu}^{-} \tilde{F}_{\nu \rho}-V_{\mathrm{bos}}\right)+\mathcal{O}\left(\frac{1}{v}\right),
$$

where $\tilde{F}_{\mu \nu}=\partial_{\mu} A_{\nu}^{+}-\partial_{\nu} A_{\mu}^{+}+i\left[A_{\mu}^{+}, A_{\nu}^{+}\right]$and $V_{\mathrm{bos}}$ is the reduction of the sextic potential of ABJM theory. As we shall discuss in the next subsection, in the reduction of the 6 -form coupling $A_{\mu}^{-}$ dependent terms are absent. Therefore, solving the equation of motion for $A_{\mu}^{-}$from (4.43) and (4.44) gives

$$
A_{\mu}^{-}=\frac{k}{4 \pi v^{2}} \epsilon_{\mu}^{\nu \rho}\left(\tilde{F}_{\nu \rho}+\mu_{2} v \lambda \frac{2 \pi}{k} P\left[\tilde{B}_{\nu \rho}\right]\right)=\frac{1}{2 g_{\mathrm{YM}} v} \epsilon_{\mu}^{\nu \rho}\left(\tilde{F}_{\nu \rho}+\frac{1}{\tilde{\lambda}} P\left[\tilde{B}_{\nu \rho}\right]\right)
$$

where

$$
P\left[\tilde{B}_{\mu \nu}\right]=\frac{1}{2}\left(\tilde{B}_{\mu \nu}+\lambda\left\langle\left\langle\tilde{B}_{\mu i} \tilde{D}_{\nu} \tilde{X}^{i}\right\rangle\right\rangle+\frac{\lambda^{2}}{3}\left\langle\left\langle\tilde{B}_{i j} \tilde{D}_{\nu} \tilde{X}^{i} \tilde{D}_{\rho} \tilde{X}^{j}\right\rangle\right\rangle\right) .
$$


Finally, inserting (4.45) into the equations (4.43) and (4.44) and rescaling the scalar field as $\tilde{X}^{i} \rightarrow \frac{\tilde{X}^{i}}{g_{\mathrm{YM}}}$, we obtain the following 10-dimensional action,

$$
\begin{aligned}
\int d^{3} x & \left\{\frac{1}{g_{\mathrm{YM}}^{2}}\left[-\tilde{D}_{\mu} \tilde{X}^{i} \tilde{D}^{\mu} \tilde{X}^{i}-\frac{1}{2}\left(\tilde{F}_{\mu \nu}+\frac{1}{\tilde{\lambda}} P\left[\tilde{B}_{\mu \nu}\right]\right)^{2}+\frac{1}{8}\left[\tilde{X}^{i}, \tilde{X}^{j}\right]^{2}\right]\right. \\
& \left.+\frac{\mu_{2}}{3 !} \epsilon^{\mu \nu \rho}\left(\tilde{C}_{\mu \nu \rho}+3 \tilde{\lambda} \tilde{C}_{\mu \nu i} \tilde{D}_{\rho} \tilde{X}^{i}+3 \tilde{\lambda}^{2} \tilde{C}_{\mu i j} \tilde{D}_{\nu} \tilde{X}^{i} \tilde{D}_{\rho} \tilde{X}^{j}+\tilde{\lambda}^{3} \tilde{C}_{i j k} \tilde{D}_{\mu} \tilde{X}^{i} \tilde{D}_{\nu} \tilde{X}^{j} \tilde{D}_{\rho} \tilde{X}^{k}\right)\right\} .
\end{aligned}
$$

Here we notice that in addition to the natural couplings of the D2-brane to the R-R 3-form fields in type IIA string theory, our WZ-type coupling (2.3) for the 3-form fields also produces the coupling between $\tilde{F}_{\mu \nu}$ and $\tilde{B}_{\mu \nu}$ in the linearized non-Abelian DBI action for D2-branes.

\subsubsection{6-form fields}

In this subsection we discuss the coupling to the 6 -form fields in the action (2.8). Later we will show that the dimensionless parameter $\tau$ we introduced in (2.8) is proportional to $1 / k$. Therefore, in the doubling scaling limit $v, k \rightarrow \infty$ with fixed $v / k$, only the terms of order $v$ or higher are nonvanishing in the 6 -form field action. To the leading order of $v$, the first term in (2.8) is reduced to

$$
\left.\epsilon^{\mu \nu \rho}\{\operatorname{Tr}\}\left(C_{\mu \nu \rho A B \bar{C}} \beta_{C}^{A B}\right)+\text { (c.c. }\right)=v i \epsilon^{\mu \nu \rho} \operatorname{Tr}\left(\tilde{C}_{\mu \nu \rho i j}\left[\tilde{X}^{i}, \tilde{X}^{j}\right]\right),
$$

where we identified the R-R 5-form fields $\tilde{C}_{\mu \nu \rho i j}$ as

$$
\begin{aligned}
& \tilde{C}_{\mu \nu \rho a 4}=-\frac{i}{2}\left(C_{\mu \nu \rho a 4 \overline{4}}-C_{\mu \nu \rho a 4 \overline{4}}^{\dagger}\right), \quad \tilde{C}_{\mu \nu \rho 4 a+4}=-\frac{1}{2}\left(C_{\mu \nu \rho a 4 \overline{4}}+C_{\mu \nu \rho a 4 \overline{4}}^{\dagger}\right), \\
& \tilde{C}_{\mu \nu \rho a b}=-\frac{i}{4}\left(C_{\mu \nu \rho a 4 \bar{b}}-C_{\mu \nu \rho a 4 \bar{b}}^{\dagger}-C_{\mu \nu \rho b 4 \bar{a}}+C_{\mu \nu \rho b 4 \bar{a}}^{\dagger}+C_{\mu \nu \rho a b \overline{4}}-C_{\mu \nu \rho a b \overline{4}}^{\dagger}\right), \\
& \tilde{C}_{\mu \nu \rho a b+4}=-\frac{1}{4}\left(C_{\mu \nu \rho a 4 \bar{b}}+C_{\mu \nu \rho a 4 \bar{b}}^{\dagger}+C_{\mu \nu \rho b 4 \bar{a}}+C_{\mu \nu \rho b 4 \bar{a}}^{\dagger}-C_{\mu \nu \rho a b \overline{4}}-C_{\mu \nu \rho a b \overline{4}}^{\dagger}\right), \\
& \tilde{C}_{\mu \nu \rho a+4 b+4}=-\frac{i}{4}\left(C_{\mu \nu \rho a 4 \bar{b}}-C_{\mu \nu \rho a 4 \bar{b}}^{\dagger}-C_{\mu \nu \rho b 4 \bar{a}}+C_{\mu \nu \rho b 4 \bar{a}}^{\dagger}-C_{\mu \nu \rho a b \overline{4}}+C_{\mu \nu \rho a b \overline{4}}^{\dagger}\right) .
\end{aligned}
$$

For the same reason as in the paragraph below (4.39), here also we have dropped the terms involving $C_{\mu \nu \rho 44 \overline{4}}$. We note the fact that $C_{\mu \nu \rho A B \bar{C}}$ are antisymmetric among the unbar indices $C_{\mu \nu \rho A B \bar{C}}=C_{\mu \nu \rho[A B] \bar{C}}$ is enough to antisymmetrize $C_{\mu \nu \rho i j}$.

Next we consider the $\lambda$-order terms in (2.8). After the breaking of the gauge symmetry these terms can be written as

$$
\begin{aligned}
& \left.\epsilon^{\mu \nu \rho}\{\operatorname{Tr}\}\left(C_{\mu \nu A B C \bar{D}} D_{\rho} Y^{A} \beta_{D}^{B C}+C_{\mu \nu A B \bar{C} \bar{D}} D_{\rho} Y_{C}^{\dagger} \beta_{D}^{A B}\right)+\text { (c.c. }\right) \\
& =\epsilon^{\mu \nu \rho} \operatorname{Tr}\left(C_{\mu \nu A B C \bar{D}}^{(1)} D_{\rho} Y^{A} \beta_{D}^{B C}+C_{\mu \nu A B C \bar{D}}^{(2)} \beta_{D}^{A B} D_{\rho} Y^{C}+C_{\mu \nu A B \bar{C} \bar{D}}^{(3)} D_{\rho} Y_{C}^{\dagger} \beta_{D}^{A B}\right. \\
& \left.\quad+C_{\mu \nu A B \bar{C} \bar{D}}^{(4)} \beta_{C}^{A B} D_{\rho} Y_{D}^{\dagger}\right)+ \text { (c.c.). }
\end{aligned}
$$


Keeping only the terms of order $v$ or higher and dropping the terms involving $C_{\mu \nu 444 \overline{4}}, C_{\mu \nu 44 \overline{4} \overline{4}}$, $C_{\mu \nu a 44 \overline{4}}, C_{\mu \nu a 4 \overline{4} \overline{4}}, \ldots$ according to the logic in (4.48), we have

$$
\begin{aligned}
\epsilon^{\mu \nu \rho}\{\operatorname{Tr}\}( & \left.C_{\mu \nu A B C \bar{D}} D_{\rho} Y^{A} \beta_{D}^{B C}+C_{\mu \nu A B \bar{C} \bar{D}} D_{\rho} Y_{C}^{\dagger} \beta_{D}^{A B}\right)+(\text { c.c. }) \\
=\epsilon^{\mu \nu \rho} \operatorname{Tr} & 2 C_{\mu \nu b a 4 \overline{4}}^{(1)} D_{\rho} Y^{b} \beta_{4}^{a 4}+2 C_{\mu \nu 4 a 4 \bar{b}}^{(1)} D_{\rho} Y^{4} \beta_{b}^{a 4}+2 C_{\mu \nu c a 4 \bar{b}}^{(1)} D_{\rho} Y^{c} \beta_{b}^{a 4} \\
& +C_{\mu \nu 4 a b \overline{4}}^{(1)} D_{\rho} Y^{4} \beta_{4}^{a b}+C_{\mu \nu c a b \overline{4}}^{(1)} D_{\rho} Y^{c} \beta_{4}^{a b}+2 C_{\mu \nu a 4 b \overline{4}}^{(2)} \beta_{4}^{a 4} D_{\rho} Y^{b} \\
& +2 C_{\mu \nu a 4 \bar{b} \bar{b}}^{(2)} \beta_{b}^{a 4} D_{\rho} Y^{4}+2 C_{\mu \nu a 4 c \bar{b}}^{(2)} \beta_{b}^{a 4} D_{\rho} Y^{c}+C_{\mu \nu a b 4 \overline{4}}^{(2)} \beta_{4}^{a b} D_{\rho} Y^{4} \\
& +C_{\mu \nu a b c \overline{4}}^{(2)} \beta_{4}^{a b} D_{\rho} Y^{c}+2 C_{\mu \nu a 4 \bar{b} \overline{4}}^{(3)} D_{\rho} Y_{b}^{\dagger} \beta_{4}^{a 4}+2 C_{\mu \nu a 4 \overline{4} \bar{b}}^{(3)} D_{\rho} Y_{4}^{\dagger} \beta_{b}^{a 4} \\
& +2 C_{\mu \nu a 4 \bar{c} \bar{b}}^{(3)} D_{\rho} Y_{c}^{\dagger} \beta_{b}^{a 4}+C_{\mu \nu a b \overline{4} \overline{4}}^{(3)} D_{\rho} Y_{4}^{\dagger} \beta_{4}^{a b}+C_{\mu \nu a b \bar{c} \overline{4}}^{(3)} D_{\rho} Y_{c}^{\dagger} \beta_{4}^{a b} \\
& +2 C_{\mu \nu a 4 \overline{4} \bar{b}}^{(4)} \beta_{4}^{a 4} D_{\rho} Y_{b}^{\dagger}+2 C_{\mu \nu a 4 \bar{b} \overline{4}}^{(4)} \beta_{b}^{a 4} D_{\rho} Y_{4}^{\dagger}+2 C_{\mu \nu a 4 \bar{b} \bar{c}}^{(4)} \beta_{b}^{a 4} D_{\rho} Y_{c}^{\dagger} \\
& \left.\left.+C_{\mu \nu a b \overline{4} \overline{4}}^{(4)} \beta_{4}^{a b} D_{\rho} Y_{4}^{\dagger}+C_{\mu \nu a b \overline{4} \bar{c} \bar{c}}^{(4)} \beta_{4}^{a b} D_{\rho} Y_{c}^{\dagger}\right)+ \text { (c.c. }\right) .
\end{aligned}
$$

These terms should produce the coupling of R-R 5-form fields to D2-branes, however substitution of (4.24) and (4.25) into (4.51) simultaneously produces unwanted terms. To eliminate the unwanted terms, we will follow the same diagnoses as in the case of 3 -form coupling. Specifically, we require that the R-R 5-form field is totally antisymmetric and the coefficients of the unwanted terms set to be zero. We also require that the products of $\tilde{D}_{\mu} \tilde{X}^{i}$ and $\left[\tilde{X}^{j}, \tilde{X}^{k}\right]$ are symmetrized. For instance, the coefficients of $\tilde{D}_{\rho} \tilde{X}^{i}\left[\tilde{X}^{j}, \tilde{X}^{k}\right]$ and $\left[\tilde{X}^{j}, \tilde{X}^{k}\right] \tilde{D}_{\rho} \tilde{X}^{i}$ terms are identified. In general, the 6-form fields $C^{(1)}, C^{(2)}, C^{(3)}$, and $C^{(4)}$, introduced in (4.50), are not necessarily antisymmetric in the global indices. However, in order to match the degrees of freedom, we can consistently impose antisymmetric property on some of 6 -form fields. In particular, we assign the antisymmetric property in the global indices $a, b, c$ to the following 6 -form fields,

$$
\begin{array}{llllll}
C_{\mu \nu b[a 4] \overline{4}}^{(1)}, & C_{\mu \nu 4 a b \overline{4}}^{(1)}, & C_{\mu \nu a b c \overline{4}}^{(1)}, & C_{\mu \nu[a 4] b \overline{4}}^{(2)}, & C_{\mu \nu a b 4 \overline{4}}^{(2)}, & C_{\mu \nu a b c \overline{4}}^{(2)} \\
C_{\mu \nu[a 4] \bar{b} \overline{4}}^{(3)}, & C_{\mu \nu a b \overline{4} \overline{4}}^{(3)}, & C_{\mu \nu a b \bar{c} \overline{4}}^{(3)}, & C_{\mu \nu[a 4] \overline{4} \bar{b}}^{(4)}, & C_{\mu \nu a b \overline{4} \overline{4}}^{(4)}, & C_{\mu \nu a b \overline{4} \bar{c}}^{(4)},
\end{array}
$$

where $[a 4]$ denotes antisymmetric in interchange of $a$ and 4. Taking these constraints into account, we obtain the following conditions for the remaining 6 -form fields:

$$
\begin{aligned}
& C_{\mu \nu 4 a b \overline{4}}^{(1)}=C_{\mu \nu a b 4 \overline{4}}^{(2)}=C_{\mu \nu a b \overline{4} \overline{4}}^{(3)}=C_{\mu \nu a b \overline{4} \overline{4},}^{(4)}, C_{\mu \nu a 4 \bar{b} \overline{4}}^{(3)}=C_{\mu \nu a 4 \overline{4} \bar{b}}^{(4)}=-C_{\mu \nu a 4 \overline{4} \bar{b}}^{(4) \dagger}, \\
& C_{\mu \nu a b 4 \overline{4}}^{(1)}=C_{\mu \nu a 4 b \overline{4}}^{(2)}=\frac{3}{4} C_{\mu \nu a b \overline{4} \overline{4}}^{(4)}+\frac{1}{4} C_{\mu \nu a b \overline{4} \overline{4} \overline{4}}^{(4) \dagger}, \quad C_{\mu \nu a 44 \bar{b}}^{(2)}+C_{\mu \nu b 4 \overline{4} \bar{a}}^{(3) \dagger}=2 C_{\mu \nu a 4 \overline{4} \bar{b},}^{(4) \dagger}, \\
& C_{\mu \nu 4 a 4 \bar{b}}^{(1)}=-2 C_{\mu \nu a 4 \overline{4} \bar{b}}^{(4)}-C_{\mu \nu b 4 \bar{a} \overline{4}}^{(4) \dagger}, \quad C_{\mu \nu a b c \overline{4}}^{(1)}=C_{\mu \nu a b c \overline{4} \overline{4}}^{(2)}, \quad C_{\mu \nu a b \bar{c} \overline{4}}^{(3)}=C_{\mu \nu a b \overline{4} \bar{c},}^{(4)}, \\
& C_{\mu \nu a b 4 \bar{c}}^{(1)}+C_{\mu \nu c 4 \bar{b} \bar{a}}^{(4) \dagger}=-C_{\mu \nu a 4 b \bar{c}}^{(2)}-C_{\mu \nu c 4 \bar{b} \bar{a}}^{(3) \dagger}=\frac{1}{2} C_{\mu \nu a b c \overline{4}}^{(2) \dagger}+\frac{3}{2} C_{\mu \nu a b \overline{4} \bar{c} \cdot}^{(4)} .
\end{aligned}
$$


Using the conditions in (4.52) and (4.53), we get

$$
\begin{gathered}
\epsilon^{\mu \nu \rho}\{\operatorname{Tr}\} \\
\left(C_{\mu \nu A B C \bar{D}} D_{\rho} Y^{A} \beta_{D}^{B C}+C_{\mu \nu A B \bar{C} \bar{D}} D_{\rho} Y_{C}^{\dagger} \beta_{D}^{A B}\right)+(\text { c.c. }) \\
=v i \epsilon^{\mu \nu \rho} \operatorname{Tr}\left(\tilde{C}_{\mu \nu i j k}\left\langle\left\langle\left[\tilde{X}^{i}, \tilde{X}^{j}\right] \tilde{D}_{\rho} \tilde{X}^{k}\right\rangle\right\rangle\right)
\end{gathered}
$$

where we have identified the R-R 5-form fields as

$$
\begin{aligned}
& \tilde{C}_{\mu \nu a b 4}=-i\left(C_{\mu \nu a b \overline{4} \overline{4}}^{(4)}+4 C_{\mu \nu a 4 \overline{4} \bar{b}}^{(4) \dagger}-C_{\mu \nu a b \overline{4} \overline{4}}^{(4) \dagger}\right), \quad \tilde{C}_{\mu \nu a 4 b+4}=-2\left(C_{\mu \nu a b \overline{4} \overline{4}}^{(4)}+C_{\mu \nu a b \overline{4} \overline{4}}^{(4) \dagger}\right), \\
& \tilde{C}_{\mu \nu 4 a+4 b+4}=i\left(C_{\mu \nu a b \overline{4} \overline{4}}^{(4)}-4 C_{\mu \nu a 4 \overline{4} \bar{b}}^{(4) \dagger}-C_{\mu \nu a b \overline{4} \overline{4}}^{(4) \dagger}\right), \quad \tilde{C}_{\mu \nu a b c}=-2 i\left(C_{\mu \nu a b \overline{4} \bar{c}}^{(4)}-C_{\mu \nu a b \overline{4} \bar{c}}^{(4) \dagger}\right), \\
& \tilde{C}_{\mu \nu a b c+4}=C_{\mu \nu a b c \overline{4}}^{(2)}+C_{\mu \nu a b c \overline{4}}^{(2) \dagger}+C_{\mu \nu a b \overline{4} \bar{c}}^{(4)}+C_{\mu \nu a b \overline{4} \bar{c} \bar{c}}^{(4) \dagger}, \\
& \tilde{C}_{\mu \nu a b+4 c+4}=i\left(C_{\mu \nu a b c \overline{4}}^{(2)}-C_{\mu \nu a b c \overline{4}}^{(2) \dagger}-C_{\mu \nu a b \overline{4} \bar{c}}^{(4)}+C_{\mu \nu a b \overline{4} \bar{c}}^{(4) \dagger}\right), \\
& \tilde{C}_{\mu \nu a+4 b+4 c+4}=2\left(C_{\mu \nu a b \overline{4} \bar{c}}^{(4)}+C_{\mu \nu a b \overline{4} \bar{c} \bar{c}}^{(4) \dagger}\right) .
\end{aligned}
$$

The next step is to consider the reduction of the $\lambda^{2}$-order couplings in (2.8). After the breakdown of the gauge symmetry, keeping only terms which are leading order in $v$, we can write these couplings as

$$
\begin{aligned}
\epsilon^{\mu \nu \rho}\{\operatorname{Tr}\}( & C_{\mu A B C D \bar{E}} D_{\nu} Y^{A} D_{\rho} Y^{B} \beta_{E}^{C D}+C_{\mu A B C \bar{D} \bar{E}} D_{\nu} Y^{A} D_{\rho} Y_{D}^{\dagger} \beta_{E}^{B C} \\
& \left.+C_{\mu A B \bar{C} \bar{D} \bar{E}} D_{\nu} Y_{C}^{\dagger} D_{\rho} Y_{D}^{\dagger} \beta_{E}^{A B}\right)+(\text { c.c. }) \\
=\epsilon^{\mu \nu \rho} \operatorname{Tr}( & C_{\mu A B C D \bar{E}}^{(1)} D_{\nu} Y^{A} \beta_{E}^{B C} D_{\rho} Y^{D}+C_{\mu A B C \bar{D} \bar{E}}^{(2)} D_{\nu} Y^{A} \beta_{D}^{B C} D_{\rho} Y_{E}^{\dagger} \\
& \left.+C_{\mu A B C \bar{D} \bar{E}}^{(3)} D_{\nu} Y_{D}^{\dagger} \beta_{E}^{A B} D_{\rho} Y^{C}+C_{\mu A B \bar{C} \bar{D} \bar{E}}^{(4)} D_{\nu} Y_{C}^{\dagger} \beta_{D}^{A B} D_{\rho} Y_{E}^{\dagger}\right)+ \text { (c.c.) } \\
& + \text { (permutations) }
\end{aligned}
$$

where 'permutations' represents the terms which can be obtained by interchanging $\beta_{C}^{A B}$ and covariant derivatives. Since the terms which are obtained by the permutations do not mix with $D Y \beta D Y$ terms, we will explicitly calculate only $D Y \beta D Y$ terms and then symmetrize the final results.

As we did previously, we keep only terms of order $v$ or higher and also drop terms involving 
6-form fields such as $C_{\mu a b 444}, C_{\mu a 4444}, C_{\mu 44444}$. Then we obtain

$$
\begin{aligned}
& \epsilon^{\mu \nu \rho} \operatorname{Tr}\left(C_{\mu A B C D \bar{E}}^{(1)} D_{\nu} Y^{A} \beta_{E}^{B C} D_{\rho} Y^{D}+C_{\mu A B C \bar{D} \bar{E}}^{(2)} D_{\nu} Y^{A} \beta_{D}^{B C} D_{\rho} Y_{E}^{\dagger}\right. \\
& \left.+C_{\mu A B C \bar{D} \bar{E}}^{(3)} D_{\nu} Y_{D}^{\dagger} \beta_{E}^{A B} D_{\rho} Y^{C}+C_{\mu A B \bar{C} \bar{D} \bar{E}}^{(4)} D_{\nu} Y_{C}^{\dagger} \beta_{D}^{A B} D_{\rho} Y_{E}^{\dagger}\right)+(\text { c.c. }) \\
& =\epsilon^{\mu \nu \rho} \operatorname{Tr}\left(2 C_{\mu b a 4 c \overline{4}}^{(1)} D_{\nu} Y^{b} \beta_{4}^{a 4} D_{\rho} Y^{c}+2 C_{\mu c a 44 \bar{b}}^{(1)} D_{\nu} Y^{c} \beta_{b}^{a 4} D_{\rho} Y^{4}+2 C_{\mu 4 a 4 c \bar{b}}^{(1)} D_{\nu} Y^{4} \beta_{b}^{a 4} D_{\rho} Y^{c}\right. \\
& +2 C_{\mu c a 4 d \bar{b}}^{(1)} D_{\nu} Y^{c} \beta_{b}^{a 4} D_{\rho} Y^{d}+C_{\mu c a b 4 \overline{4}}^{(1)} D_{\nu} Y^{c} \beta_{4}^{a b} D_{\rho} Y^{4}+C_{\mu 4 a b c \overline{4}}^{(1)} D_{\nu} Y^{4} \beta^{a b}{ }_{4} D_{\rho} Y^{c} \\
& +C_{\mu 4 a b c \overline{4}}^{(1)} D_{\nu} Y^{c} \beta_{4}^{a b} D_{\rho} Y^{d}+2 C_{\mu b a 4 \overline{4} \bar{c}}^{(2)} D_{\nu} Y^{b} \beta_{4}^{a 4} D_{\rho} Y_{c}^{\dagger}+2 C_{\mu c a 4 \bar{b} \overline{4}}^{(2)} D_{\nu} Y^{c} \beta_{b}^{a 4} D_{\rho} Y_{d}^{\dagger} \\
& +2 C_{\mu 4 a 4 \bar{b} \bar{c}}^{(2)} D_{\nu} Y^{4} \beta_{b}^{a 4} D_{\rho} Y_{c}^{\dagger}+2 C_{\mu c a 4 \bar{b} \bar{d}}^{(2)} D_{\nu} Y^{c} \beta_{b}^{a 4} D_{\rho} Y_{d}^{\dagger}+C_{\mu c a b \overline{4} \overline{4}}^{(2)} D_{\nu} Y^{c} \beta_{4}^{a b} D_{\rho} Y_{d}^{\dagger} \\
& +C_{\mu 4 a b \overline{4} \bar{c}}^{(2)} D_{\nu} Y^{4} \beta_{4}^{a b} D_{\rho} Y_{c}^{\dagger}+C_{\mu c a b \overline{4} \bar{d}}^{(2)} D_{\nu} Y^{c} \beta_{4}^{a b} D_{\rho} Y_{d}^{\dagger}+2 C_{\mu a 4 c \bar{b} \overline{4}}^{(3)} D_{\nu} Y_{b}^{\dagger} \beta_{4}^{a 4} D_{\rho} Y^{c} \\
& +2 C_{\mu a 44 \bar{c} \bar{b}}^{(3)} D_{\nu} Y_{c}^{\dagger} \beta_{b}^{a 4} D_{\rho} Y^{4}+2 C_{\mu a 4 c \overline{4} \bar{b}}^{(3)} D_{\nu} Y_{4}^{\dagger} \beta_{b}^{a 4} D_{\rho} Y^{c}+2 C_{\mu a 4 d \bar{c} \bar{b}}^{(3)} D_{\nu} Y_{c}^{\dagger} \beta_{b}^{a 4} D_{\rho} Y^{d} \\
& +C_{\mu a b 4 \bar{c} \overline{4}}^{(3)} D_{\nu} Y_{c}^{\dagger} \beta^{a b} D_{\rho} Y^{4}+C_{\mu a b c \overline{4} \overline{4}}^{(3)} D_{\nu} Y_{4}^{\dagger} \beta_{4}^{a b} D_{\rho} Y^{c}+C_{\mu a b d \bar{c} \overline{4}}^{(3)} D_{\nu} Y_{c}^{\dagger} \beta_{4}^{a b} D_{\rho} Y^{d} \\
& +2 C_{\mu a 4 \bar{c} \bar{b} \overline{4}}^{(4)} D_{\nu} Y_{c}^{\dagger} \beta_{b}^{a 4} D_{\rho} Y_{d}^{\dagger}+2 C_{\mu a 4 \bar{b} \overline{4} \bar{c}}^{(4)} D_{\nu} Y_{b}^{\dagger} \beta_{4}^{a 4} D_{\rho} Y_{c}^{\dagger}+2 C_{\mu a 4 \overline{4} \bar{b} \bar{c}}^{(4)} D_{\nu} Y_{4}^{\dagger} \beta_{b}^{a 4} D_{\rho} Y_{c}^{\dagger} \\
& +2 C_{\mu a 4 \bar{c} \bar{b} \bar{d}}^{(4)} D_{\nu} Y_{c}^{\dagger} \beta_{b}^{a 4} D_{\rho} Y_{d}^{\dagger}+C_{\mu a b \overline{4} \overline{4} \bar{c}}^{(4)} D_{\nu} Y_{4}^{\dagger} \beta_{4}^{a b} D_{\rho} Y_{c}^{\dagger}+C_{\mu a b \bar{c} \bar{c} \bar{c}}^{(4)} D_{\nu} Y_{c}^{\dagger} \beta^{a b}{ }_{4} D_{\rho} Y_{d}^{\dagger} \\
& \left.+C_{\mu a b \bar{c} \overline{4} \bar{d}}^{(4)} D_{\nu} Y_{c}^{\dagger} \beta^{a b} D_{\rho} Y_{d}^{\dagger}\right)+ \text { (c.c.). }
\end{aligned}
$$

Following the argument in paragraph after (4.51) we impose antisymmetric property to the global indices, $a, b, c, d$, for the following 6-form fields,

$$
\begin{aligned}
& C_{\mu c a b 4 \overline{4}}^{(1)}, C_{\mu c[a 4] b \overline{4}}^{(1)}, C_{\mu 4 a b c \overline{4}}^{(1)}, C_{\mu c a b \overline{4} \overline{4}}^{(2)}, C_{\mu b[a 4] \overline{4} \bar{c}}^{(2)}, C_{\mu 4 a b \overline{4} \bar{c}}^{(2)}, C_{\mu a b c \overline{4} \overline{4}}^{(3)}, C_{\mu a b 4 \bar{c} \overline{4}}^{(3)}, \\
& C_{\mu[a 4] b \bar{c} \overline{4}}^{(3)}, C_{\mu a b \bar{c} \overline{4} \overline{4}}^{(4)}, C_{\mu a b \overline{4} \overline{4} \bar{c}}^{(4)}, C_{\mu[a 4] \bar{b} \overline{4} \bar{c}}^{(4)}, C_{\mu c a b d \overline{4}}^{(1)}, C_{\mu c a b \overline{4} \bar{d} \bar{d}}^{(2)}, C_{\mu a b d \bar{c} \overline{4}}^{(3)}, C_{\mu a b \bar{c} \overline{4} \bar{d}}^{(4)}
\end{aligned}
$$

With theses constraints the remaining 6-form fields satisfy:

$$
\begin{aligned}
& C_{\mu a b c \overline{4} \overline{4}}^{(3)}=-C_{\mu c a b \overline{4} \overline{4}}^{(2)}=C_{\mu 4 a b c \overline{4}}^{(1)}=-C_{\mu c a b 4 \overline{4}}^{(1)}, \\
& C_{\mu a 4 \bar{b} \overline{4} \bar{c}}^{(4)}=C_{\mu a 4 b \bar{c} \overline{4}}^{(3) \dagger}=-C_{\mu b a 4 \overline{4} \bar{c}}^{(2) \dagger}=2 C_{\mu c a b 4 \overline{4}}^{(1)}-3 C_{\mu c a 4 b \overline{4}}^{(1)}, \\
& C_{\mu a b \bar{c} \overline{4} \overline{4}}^{(4)}=-C_{\mu a b \overline{4} \overline{4} \bar{c}}^{(4)}=C_{\mu a b 4 \bar{c} \overline{4}}^{(3)}=-C_{\mu 4 a b \overline{4} \bar{c}}^{(2)}=-3 C_{\mu c a b 4 \overline{4}}^{(1) \dagger}+4 C_{\mu c a 4 b \overline{4}}^{(1) \dagger}, \\
& C_{\mu 4 a 4 \bar{b} \bar{c} \bar{c}}^{(2)}-C_{\mu a b 4 \bar{c} \overline{4}}^{(2) \dagger}=C_{\mu a 4 \overline{4} \bar{b} \bar{c} \bar{c}}^{(4)}-C_{\mu a b 44 \bar{c}}^{(1) \dagger}=C_{\mu a 44 \bar{b} \bar{c}}^{(3)}-C_{\mu c 4 b \overline{4} \bar{a}}^{(3) \dagger} \\
& =C_{\mu a 4 \bar{b} \bar{b} \bar{c} \overline{4}}^{(4)}-C_{\mu 4 c 4 b \bar{a}}^{(1) \dagger}=-4 C_{\mu c a b 4 \overline{4}}^{(1)}+6 C_{\mu c a 4 b \overline{4} \bar{c}}^{(1)}, \\
& C_{\mu a b d \bar{c} \overline{4}}^{(3)}=C_{\mu a b d \bar{c} \overline{4}}^{(3) \dagger}=-C_{\mu c a b \overline{4} \bar{d}}^{(2)}=-C_{\mu c a b \overline{4} \bar{d},}^{(2) \dagger}, \\
& C_{\mu b a 4 \bar{c} \bar{d}}^{(2)}-C_{\mu b c 4 \bar{a} \bar{d}}^{(2) \dagger}=-C_{\mu d 4 a \bar{b} \bar{c} \bar{c}}^{(3)}+C_{\mu c 4 b \bar{a} \bar{d}}^{(3) \dagger}=\frac{1}{4} C_{\mu c a b d \overline{4}}^{(1)}-\frac{1}{4} C_{\mu c a b d \overline{4}}^{(1) \dagger}, \\
& C_{\mu a b \bar{c} \bar{c} \bar{d}}^{(4)}=-\frac{1}{6} C_{\mu c a b d \overline{4}}^{(1)}-\frac{1}{6} C_{\mu c a b d \overline{4}}^{(1) \dagger}, \\
& C_{\mu a 4 \bar{b} \bar{c} \bar{d} \bar{d}}^{(4)}=C_{\mu d c 4 a \bar{b}}^{(1) \dagger}+2 C_{\mu c a b \overline{4} \bar{d} .}^{(2)} .
\end{aligned}
$$


Under these conditions (4.57) is reduced to

$$
\begin{aligned}
\epsilon^{\mu \nu \rho} & \operatorname{Tr}\left(C_{\mu A B C D \bar{E}}^{(1)} D_{\nu} Y^{A} \beta_{E}^{B C} D_{\rho} Y^{D}+C_{\mu A B C \bar{D} \bar{E}}^{(2)} D_{\nu} Y^{A} \beta_{D}^{B C} D_{\rho} Y_{E}^{\dagger}\right. \\
& \left.+C_{\mu A B C \bar{D} \bar{E}}^{(3)} D_{\nu} Y_{D}^{\dagger} \beta_{E}^{A B} D_{\rho} Y^{C}+C_{\mu A B \bar{C} \bar{D} \bar{E}}^{(4)} D_{\nu} Y_{C}^{\dagger} \beta_{D}^{A B} D_{\rho} Y_{E}^{\dagger}\right)+(\text { c.c. }) \\
& =v i \epsilon^{\mu \nu \rho} \operatorname{Tr}\left(\tilde{C}_{\mu i j k l} D_{\nu} \tilde{X}^{i}\left[\tilde{X}^{j}, \tilde{X}^{k}\right] D_{\rho} \tilde{X}^{l}\right),
\end{aligned}
$$

where we identified the R-R 5-form fields as

$$
\begin{aligned}
& \tilde{C}_{\mu a b c 4}=-2 i\left(3 C_{\mu c a \overline{4} b 4}^{(1)}-3 C_{\mu c a b 4 \overline{4}}^{(1) \dagger}-4 C_{\mu c a 4 b \overline{4}}^{(1)}+4 C_{\mu c a 4 b \overline{4}}^{(1) \dagger}\right), \\
& \tilde{C}_{\mu a b 4 c+4}=2\left(C_{\mu c a b 4 \overline{4}}^{(1)}+C_{\mu c a b 4 \overline{4}}^{(1)}-2 C_{\mu c a 4 b \overline{4}}^{(1)}-2 C_{\mu c a 4 b \overline{4}}^{(1)}\right), \\
& \tilde{C}_{\mu a 4 b+4 c+4}=-2 i\left(C_{\mu c a b 4 \overline{4}}^{(1)}-C_{\mu c a b 4 \overline{4}}^{(1) \dagger}-2 C_{\mu c a 4 b \overline{4}}^{(1)}+2 C_{\mu c a b b \overline{4}}^{(1) \dagger}\right), \\
& \tilde{C}_{\mu 4 a+4 b+4 c+4}=2\left(3 C_{\mu c a b 4 \overline{4}}^{(1)}+3 C_{\mu c a b 4 \overline{4}}^{(1) \dagger}-4 C_{\mu c a 4 b \overline{4}}^{(1)}-4 C_{\mu c a 4 b \overline{4}}^{(1) \dagger}\right), \\
& \tilde{C}_{\mu a b c d+4}=\frac{1}{3}\left(C_{\mu c a b d \overline{4}}^{(1)}+C_{\mu c a b d \overline{4}}^{(1) \dagger}+6 C_{\mu c a b \overline{4} \bar{d} \bar{d}}^{(2)}\right), \\
& \tilde{C}_{\mu a b c+4 d+4}=\frac{i}{2}\left(C_{\mu c a b d \overline{4} \overline{4}}^{(1)}-C_{\mu c a b d \overline{4}}^{(1) \dagger}\right), \\
& \tilde{C}_{\mu a b+4 c+4 d+4}=-\frac{1}{3}\left(C_{\mu c a b d \overline{4}}^{(1)}+C_{\mu c a b d \overline{4}}^{(1) \dagger}-6 C_{\mu c a b \overline{4} \bar{d}}^{(2)}\right) .
\end{aligned}
$$

Finally, including the 'permutations' terms in (4.56) and identifying the R-R 5-form fields for all the terms related by permutation of the anticommutator $\left[\tilde{X}^{i}, \tilde{X}^{j}\right]$ and the covariant derivative $\tilde{D}_{\mu} \tilde{X}^{k}$, we obtain the following symmetrized result,

$$
\begin{aligned}
\epsilon^{\mu \nu \rho} \operatorname{Tr} & \left(C_{\mu A B C D \bar{E}}^{(1)} D_{\nu} Y^{A} \beta_{E}^{B C} D_{\rho} Y^{D}+C_{\mu A B C \bar{D} \bar{E}}^{(2)} D_{\nu} Y^{A} \beta_{D}^{B C} D_{\rho} Y_{E}^{\dagger}+C_{\mu A B C \bar{D} \bar{E}}^{(3)} D_{\nu} Y_{D}^{\dagger} \beta_{E}^{A B} D_{\rho} Y^{C}\right. \\
& \left.+C_{\mu A B \bar{C} \bar{D} \bar{E}}^{(4)} D_{\nu} Y_{C}^{\dagger} \beta_{D}^{A B} D_{\rho} Y_{E}^{\dagger}\right)+(\text { c.c. })+(\text { permutations }) \\
= & v i \epsilon^{\mu \nu \rho} \operatorname{Tr}\left(\tilde{C}_{\mu i j k l}\left\langle\left\langle\left[\tilde{X}^{i}, \tilde{X}^{j}\right] D_{\nu} \tilde{X}^{k} D_{\rho} \tilde{X}^{l}\right\rangle\right\rangle\right) .
\end{aligned}
$$

It remains to carry out the dimensional reduction of the $\lambda^{3}$-order terms in (2.8). Due to a huge number of possible terms, for this order we could not be able to carry out the reduction procedure. However, looking at the results for the $\lambda$ and $\lambda^{2}$-order terms, we can easily expect that in the compactification limit the $\lambda^{3}$-order terms will produce the following 10-dimensional WZ-type coupling,

$$
v i \epsilon^{\mu \nu \rho} \operatorname{Tr}\left(\tilde{C}_{i j k l m}\left\langle\left\langle\left[\tilde{X}^{i}, \tilde{X}^{j}\right] \tilde{D}_{\mu} \tilde{X}^{k} \tilde{D}_{\nu} \tilde{X}^{l} D_{\rho} \tilde{X}^{m}\right\rangle\right\rangle\right) .
$$

The relations between the R-R 5 form $\tilde{C}_{i j k l m}$ and the 6 -form fields need explicit calculation.

Substituting the relations (4.48), (4.54), (4.62), and (4.63) into the 6-form field coupling (2.8) and choosing the dimensionless parameter $\tau$ as $-\pi / k$, we obtain the expected WZ-type coupling 
for R-R 5-form fields in type IIA string theory,

$$
\begin{aligned}
S_{\tilde{C}}^{(5)}= & -\frac{\mu_{2} \tilde{\lambda}}{2} \int d^{3} x \epsilon^{\mu \nu \rho} \frac{1}{3 !}\left(i \tilde{C}_{\mu \nu \rho i j}\left[\tilde{X}^{i}, \tilde{X}^{j}\right]+3 i \tilde{\lambda} \tilde{C}_{\mu \nu i j k}\left\langle\left\langle\left[\tilde{X}^{i}, \tilde{X}^{j}\right] \tilde{D}_{\rho} \tilde{X}^{k}\right\rangle\right\rangle\right. \\
& \left.+3 i \tilde{\lambda}^{2} \tilde{C}_{\mu i j k l}\left\langle\left\langle\left[\tilde{X}^{i}, \tilde{X}^{j}\right] \tilde{D}_{\nu} \tilde{X}^{k} \tilde{D}_{\rho} \tilde{X}^{l}\right\rangle\right\rangle+i \tilde{\lambda}^{3} \tilde{C}_{i j k l m}\left\langle\left\langle\left[\tilde{X}^{i}, \tilde{X}^{j}\right] \tilde{D}_{\mu} \tilde{X}^{k} \tilde{D}_{\nu} \tilde{X}^{l} \tilde{D}_{\rho} \tilde{X}^{m}\right\rangle\right\rangle\right) .
\end{aligned}
$$

\section{Comments on Mass Deformations}

The supersymmetry preserving mass deformation of the ABJM theory was introduced in Refs. 21, 22]. There are several methods to obtain the mass-deformed ABJM theory, such as $\mathcal{N}=1$ superfield formalism [21], $D$-term and $F$-term deformations in $\mathcal{N}=2$ superfield formalism [22]. These different versions of mass-deformed ABJM theory are equivalent since they are connected by field redefinitions [23]. A different intriguing question is to identify possible source of the supersymmetry preserving mass deformation. In this section we will address this question for the quartic mass deformation.

The quadratic and quartic terms of the mass deformation are given by

$$
S_{\mu}=\mu^{2} \int d^{3} x \operatorname{Tr}\left(Y^{A} Y_{A}^{\dagger}\right)+\frac{4 \pi \mu}{k} \int d^{3} x \operatorname{Tr}\left(M_{B}^{C} Y^{A} Y_{A}^{\dagger} Y^{B} Y_{C}^{\dagger}-M_{C}^{B} Y_{A}^{\dagger} Y^{A} Y_{B}^{\dagger} Y^{C}\right)
$$

where $M_{A}^{B}=\operatorname{diag}(1,1,-1,-1)$. In our present conventions these deformations can be written as

$$
\left.S_{\mu}=\mu^{2} \int d^{3} x \operatorname{Tr}\left(Y^{A} Y_{A}^{\dagger}\right)-\frac{2 \pi \mu}{k} \int d^{3} x \operatorname{Tr}\left(T_{A B \bar{C} \bar{D}} Y_{D}^{\dagger} \beta_{C}^{A B}\right)+\text { (c.c. }\right),
$$

where $T_{A B \bar{C} \bar{D}}=M_{A}{ }^{D} \delta_{B}^{C}-M_{B}{ }^{D} \delta_{A}{ }^{C}$. With an appropriate choice of the 6 -form field, it is possible to identify the quartic mass deformation action in (5.2) with part of our WZ-type coupling in (2.8). To that end let us consider the first term 4 in (2.8),

$$
\left.S_{\mu}^{(6)}=\mu_{2}^{\prime} \int d^{3} x \frac{1}{3 !} \epsilon^{\mu \nu \rho} \operatorname{Tr}\left(C_{\mu \nu \rho A B \bar{C}} \beta_{C}^{A B}\right)+\text { (c.c. }\right),
$$

where for this term the $\{\operatorname{Tr}\}$ in (2.8) is equivalent to ordinary trace. In order to identify the quartic mass deformation with (5.3) the 6-form field should take the following structure,

$$
C_{\mu \nu \rho A B \bar{C}}=-\frac{2 \mu}{\lambda \mu_{2}} \epsilon_{\mu \nu \rho} T_{A B \bar{C} \bar{D}} Y_{D}^{\dagger}, \quad C_{\mu \nu \rho A B \bar{C}}^{\dagger}=-\frac{2 \mu}{\lambda \mu_{2}} \epsilon_{\mu \nu \rho} T_{A B \bar{C} \bar{D}}^{\dagger} Y^{D} .
$$

\footnotetext{
${ }^{4}$ In [11], the authors considered the second and the third terms in (2.8) instead of the first term. This is just a different choice of gauge, which results in the same constant field strength.
} 
Since $T_{A B \bar{C} \bar{D}}$ is real, the constant 7 -form field strength corresponding to both these 6 -form fields are the same,

$$
F_{\mu \nu \rho A B \bar{C} \bar{D}}=F_{\mu \nu \rho A B \bar{C} \bar{D}}^{\dagger}=-\frac{2 \mu}{\lambda^{2} \mu_{2}} \epsilon_{\mu \nu \rho} T_{A B \bar{C} \bar{D}}
$$

In the eight-dimensional transverse space this can be viewed as turning on a constant (anti)-self dual 4-form field strength,

$$
F_{A B \bar{C} \bar{D}}=-\frac{2 \mu}{\lambda^{2} \mu_{2}} T_{A B \bar{C} \bar{D}}
$$

Therefore, in order to get the supersymmetry preserving quartic mass deformation of the ABJM theory we need to turn on the constant 4-form field strengths in (5.6) in the direction transverse to the stack of parallel multiple M2-branes.5 This is in accordance with Refs. 24, 25, 11, 26, which state that the mass-deformed ABJM action comes from a background (anti)self dual 4-form flux in the eight-dimensional transverse space. The quadratic mass-deformed term in (5.2) is originated from the backreaction of the background metric in the presence of the 4-form flux [11].

\section{Conclusion and Discussion}

In this work we discussed the coupling of multiple M2-branes to the background 3- and 6-form fields in 11-dimensional supergravity. We proposed a general gauge-invariant WZ-type coupling in the ABJM theory with $\mathrm{U}(N) \times \mathrm{U}(N)$ gauge group. The fundamental building blocks of the coupling are the 3- and 6-form gauge fields, the covariant derivative $D_{\mu} Y^{A}$, the 3-commutator $\beta_{C}^{A B}$, and their complex conjugates. We assumed that the form fields depend on the transverse scalars $Y^{A}$ and their complex conjugates. Therefore, the form fields carry gauge indices according to their dependence on $Y^{A}$ and $Y_{A}^{\dagger}$ to guarantee gauge invariance which allows both single and multi traces. Since the multi-trace terms after the MP Higgsing cannot be identified with any WZ-type couplings in type IIA string theory, we did not include those terms from the beginning. We tested our proposal for the WZ-type coupling through several consistency checks.

We compared our WZ-type coupling with the known coupling for a single M2-brane. In this case the gauge group is $\mathrm{U}(1) \times \mathrm{U}(1)$. We showed that our coupling completely matches the well known effective action of a single M2-brane coupled to 3-form fields. Then we carried out the MP Higgsing procedure for our WZ-type coupling including the bosonic part of the original ABJM action with $\mathrm{U}(N) \times \mathrm{U}(N)$ gauge symmetry. We obtained the symmetrized WZ-type coupling for the R-R 3- and 5-form fields with the gauge field strength turned off [7]. The MP Higgsing

\footnotetext{
${ }^{5}$ Turning on the other components of the form fields will break the supersymmetry, partially or totally.
} 
procedure alone is not enough to reproduce all the expected WZ-type couplings in type IIA string theory. Therefore, we imposed some constraints on the 11-dimensional form fields, in order

- to make the 10-dimensional form fields antisymmetric,

- to obtain a 10-dimensional WZ-type coupling which is symmetrized with respect to interchange of the covariant derivatives $\left(\tilde{D}_{\mu} \tilde{X}^{i}\right)$ and the commutators $\left(\left[\tilde{X}^{j}, \tilde{X}^{k}\right]\right)$, and

- to make disappear quadratic terms in the auxiliary gauge field $A_{\mu}^{-}$in 3 -form coupling and linear terms in $A_{\mu}^{-}$in 6-form coupling, which are absent in WZ-type coupling in type IIA string theory.

We have also showed that, in addition to the WZ-type coupling for the R-R 3- and 5-form fields, the reduction procedure also produces correctly the linearized version of DBI action for the gauge field strength $\tilde{F}_{\mu \nu}$ of the dynamical gauge field $A_{\mu}^{+}$and the NS-NS 2-form field $\tilde{B}_{\mu \nu}$. Finally, we tested the validity of our proposal by establishing the relation between the WZ-type coupling for the 6-form field and the supersymmetry preserving quartic term of the mass-deformed action in ABJM theory with $\mathrm{U}(N) \times \mathrm{U}(N)$ gauge symmetry.

A few comments about our construction are in order. Though the 10-dimensional WZ-type coupling is restricted to the linear terms in form fields, it contains an exponential form of the coupling of the NS-NS 2-form field. Since the NS-NS 2-form field comes from compactification of the 3-form field in M-theory, it seems natural to include nonlinear terms which deserves further investigations. In addition to the fundamental building blocks of our WZ-type coupling listed above, we can add gauge field strengths for $A_{\mu}$ and $\hat{A}_{\mu}$ and the monopole operator, which changes bifundamental representation to antibifundamental and vice versa for $k=1,2$ [27]. With these extensions we may include extra gauge-invariant terms to our WZ-type couplings.

\section{Acknowledgements}

The authors would like to appreciate the informative discussions with Jaemo Park. This work was supported by the Korea Research Foundation Grant funded by the Korean Government with grant number KRF-2008-313-C00170 (Y.K.), 2009-0073775 (O.K.), and 2009-0077423 (D.D.T.). This work was also supported by the National Research Foundation of Korea (NRF) grant funded by the Korean Government (MEST) (No. 2009-0062869) through Astrophysical Research Center for the Structure and Evolution of the Cosmos (ARCSEC) (Y.K.), and by Mid-career Researcher Program through the National Research Foundation of Korea(NRF) grant funded by the Korea government(MEST)(No. 2009-0084601) (H.N.). 


\section{A Comments on Single Trace Terms}

In our construction of the WZ-type actions in (2.3) and (2.8) the 3- and 6-form fields are arbitrary functions of the transverse complex scalar fields except that every term in the actions

- should contain equal number of bifundamental and antibifundamental fields to guarantee gauge invariance,

- and should be single trace.

After the MP Higgsing procedure, this arbitrariness results in terms which are absent in the known WZ-type action of in type IIA string theory. In this appendix we shall show how to overcome this problem by imposing more constraints on the functional dependence of the form fields on the complex scalar fields.

Let us consider the following term in (2.3),

$$
\{\operatorname{Tr}\}\left(C_{\mu A \bar{B}} D_{\nu} Y^{A} D_{\rho} Y_{B}^{\dagger}\right) .
$$

For clear presentation we drop the worldvolume and global indices from now on. Imposing the single traceness constraint on (A.1) we have

$$
\{\operatorname{Tr}\}\left(C D Y D Y^{\dagger}\right)=C_{a \hat{b}}^{\hat{a} b}(D Y)_{\hat{a}}^{a}\left(D Y^{\dagger}\right)_{b}^{\hat{b}}=\operatorname{Tr}\left(H D Y^{A} I D Y^{\dagger}\right),
$$

where $C_{a \hat{b}}^{\hat{a} b}=H_{\hat{b}}^{\hat{a}} I_{a}^{b}$, and both $H$ and $I$ should depend on the transverse scalar fields to guarantee the $\mathrm{U}(N) \times \mathrm{U}(N)$ gauge invariance. If we turn on the vev for the scalar fields, $Y^{A} \rightarrow \frac{v}{2} \delta^{A 4}+Y^{A}$, it breaks the $\mathrm{U}(N) \times \mathrm{U}(N)$ gauge symmetry to a $\mathrm{U}(N)$ and then both the bifundamental and antibifundamental fields follow adjoint representation. For those adjoint fields the indices $a, b, \cdots$ and $\hat{a}, \hat{b}, \cdots$ are indistinguishable so that the matrices $H$ and $I$ are expressed as

$$
\begin{aligned}
H^{a}{ }_{b} & =h_{0} \delta_{b}^{a}+h_{1} \tilde{Y}_{b}^{a}+h_{2}(\tilde{Y} \tilde{Y})^{a}{ }_{b}+\cdots, \\
I^{a}{ }_{b} & =i_{0} \delta_{b}^{a}+i_{1} \tilde{Y}_{b}^{a}+i_{2}(\tilde{Y} \tilde{Y})^{a}{ }_{b}+\cdots,
\end{aligned}
$$

where $h_{i}$ and $i_{i}$ are the coefficients which can depend on the parameters $v, k$, and $l_{\mathrm{P}}$, and $\tilde{Y}$ is either $Y$ or $Y^{\dagger}$. Substituting (A.3) in (A.2), we have

$$
\operatorname{Tr}\left(H D Y^{A} I D Y^{\dagger}\right)=\operatorname{Tr}\left(C^{(1)} D Y D Y^{\dagger}+C^{(2)} D Y^{\dagger} D Y+\mathcal{H} D Y \mathcal{I} D Y^{\dagger}\right)
$$

where

$$
\begin{aligned}
C^{(1)} & =\left(h_{0}+h_{1} \tilde{Y}+h_{2} \tilde{Y} \tilde{Y}+\cdots\right) i_{0}, \\
C^{(2)} & =\left(i_{0}+i_{1} \tilde{Y}+i_{2} \tilde{Y} \tilde{Y}+\cdots\right) h_{0}, \\
\mathcal{H} & =h_{1} \tilde{Y}+h_{2} \tilde{Y} \tilde{Y}+\cdots, \\
\mathcal{I} & =i_{1} \tilde{Y}+i_{2} \tilde{Y} \tilde{Y}+\cdots .
\end{aligned}
$$


In our reduction procedure in section 4 we have dropped the last term in (A.4). This is justified if we restrict the form fields in string theory be at most linear in the scalar fields. In generic setup we can realize the last term in (A.4) can appear. Therefore, in order to reproduce the known WZ-type coupling with arbitrary dependence on the transverse scalars in type IIA string theory, we should find an appropriate condition on the form field couplings in M-theory to forbid the last term in (A.4) and all the other terms of this kind.

\section{References}

[1] J. Bagger and N. Lambert, "Modeling multiple M2's," Phys. Rev. D 75, 045020 (2007) arXiv:hep-th/0611108]; "Gauge Symmetry and Supersymmetry of Multiple M2-Branes," Phys. Rev. D 77, 065008 (2008) arXiv:0711.0955 [hep-th]]; "Comments On Multiple M2branes," JHEP 0802, 105 (2008) arXiv:0712.3738 [hep-th]].

[2] A. Gustavsson, "Algebraic structures on parallel M2-branes," Nucl. Phys. B 811, 66 (2009) arXiv:0709.1260 [hep-th]].

[3] O. Aharony, O. Bergman, D. L. Jafferis and J. Maldacena, "N=6 superconformal ChernSimons-matter theories, M2-branes and their gravity duals," JHEP 0810, 091 (2008) arXiv:0806.1218 [hep-th]].

[4] M. Van Raamsdonk, "Comments on the Bagger-Lambert theory and multiple M2-branes," JHEP 0805, 105 (2008) [arXiv:0803.3803 [hep-th]].

[5] M. Li, "Boundary States of D-Branes and Dy-Strings," Nucl. Phys. B 460, 351 (1996) arXiv:hep-th/9510161.

[6] M. R. Douglas, "Branes within branes," arXiv:hep-th/9512077;

M. B. Green, J. A. Harvey and G. W. Moore, "I-brane inflow and anomalous couplings on D-branes," Class. Quant. Grav. 14, 47 (1997) arXiv:hep-th/9605033].

[7] R. C. Myers, "Dielectric-branes," JHEP 9912, 022 (1999) arXiv:hep-th/9910053].

[8] M. Li and T. Wang, "M2-branes Coupled to Antisymmetric Fluxes," JHEP 0807, 093 (2008) arXiv:0805.3427 [hep-th]].

[9] M. A. Ganjali, "On Dielectric Membranes," JHEP 0905, 047 (2009) arXiv:0901.2642 [hepth]]. 
[10] Y. Kim, O. K. Kwon, H. Nakajima and D. D. Tolla, "Coupling between M2-branes and Form Fields," JHEP 0910, 022 (2009) [arXiv:0905.4840 [hep-th]].

[11] N. Lambert and P. Richmond, "M2-Branes and Background Fields," JHEP 0910 (2009) 084 arXiv:0908.2896 [hep-th]].

[12] S. Sasaki, "On Non-linear Action for Gauged M2-brane," JHEP 1002 (2010) 039 arXiv:0912.0903 [hep-th]].

[13] S. Mukhi and C. Papageorgakis, "M2 to D2," JHEP 0805, 085 (2008) arXiv:0803.3218 [hep-th]].

[14] B. Ezhuthachan, S. Mukhi and C. Papageorgakis, "The Power of the Higgs Mechanism: Higher-Derivative BLG Theories," JHEP 0904, 101 (2009) [arXiv:0903.0003 [hep-th]].

[15] M. R. Garousi and R. C. Myers, "World-volume interactions on D-branes," Nucl. Phys. B 542, 73 (1999) arXiv:hep-th/9809100.

[16] J. Bagger and N. Lambert, "Three-Algebras and N=6 Chern-Simons Gauge Theories," Phys. Rev. D 79, 025002 (2009) arXiv:0807.0163 [hep-th]].

[17] C. Ciocarlie, "On the gauge invariance of the Chern-Simons action for N D-branes," JHEP 0107, 028 (2001) arXiv:hep-th/0105253.

[18] J. Adam, J. Gheerardyn, B. Janssen and Y. Lozano, "The gauge invariance of the non-Abelian Chern-Simons action for D-branes revisited," Phys. Lett. B 589, 59 (2004) arXiv:hep-th/0312264.

[19] Y. Kim, O-K. Kwon, H. Nakajima, and D. D. Tolla, in preparation.

[20] E. Bergshoeff, E. Sezgin and P. K. Townsend, "Supermembranes and eleven-dimensional supergravity," Phys. Lett. B 189, 75 (1987).

[21] K. Hosomichi, K. M. Lee, S. Lee, S. Lee and J. Park, "N=5,6 Superconformal Chern-Simons Theories and M2-branes on Orbifolds," JHEP 0809, 002 (2008) [arXiv:0806.4977 [hep-th]].

[22] J. Gomis, D. Rodriguez-Gomez, M. Van Raamsdonk and H. Verlinde, "A Massive Study of M2-brane Proposals," JHEP 0809, 113 (2008) [arXiv:0807.1074 [hep-th]].

[23] C. Kim, Y. Kim, O. K. Kwon and H. Nakajima, "Vortex-type Half-BPS Solitons in ABJM Theory," Phys. Rev. D 80, 045013 (2009) [arXiv:0905.1759 [hep-th]]. 
[24] I. Bena, "The M-theory dual of a 3 dimensional theory with reduced supersymmetry," Phys. Rev. D 62 (2000) 126006 arXiv:hep-th/0004142].

[25] H. Lin, O. Lunin and J. M. Maldacena, "Bubbling AdS space and 1/2 BPS geometries," JHEP 0410, 025 (2004) arXiv:hep-th/0409174].

[26] H. C. Kim and S. Kim, "Supersymmetric vacua of mass-deformed M2-brane theory," Nucl. Phys. B 839, 96 (2010) arXiv:1001.3153 [hep-th]].

[27] A. Gustavsson and S. J. Rey, "Enhanced N=8 Supersymmetry of ABJM Theory on R(8) and $\mathrm{R}(8) / \mathrm{Z}(2)$, , arXiv:0906.3568 [hep-th] ;

O. K. Kwon, P. Oh and J. Sohn, "Notes on Supersymmetry Enhancement of ABJM Theory," JHEP 0908, 093 (2009) arXiv:0906.4333 [hep-th]] ;

D. Bashkirov and A. Kapustin, "Supersymmetry enhancement by monopole operators," arXiv:1007.4861 [hep-th] ;

H. Samtleben and R. Wimmer, "N=6 Superspace Constraints, SUSY Enhancement and Monopole Operators," arXiv:1008.2739 [hep-th]. 